\title{
A unidirectional on-chip photonic interface for superconducting circuits
}

\author{
P.-O. Guimond ${ }^{1,2 凶}$, B. Vermersch ${ }^{1,2,3}$, M. L. Juan ${ }^{2,4}$, A. Sharafiev ${ }^{2,4}$, G. Kirchmair ${ }^{2,4}$ and P. Zoller (D) ${ }^{1,2}$
}

We propose and analyze a passive architecture for realizing on-chip, scalable cascaded quantum devices. In contrast to standard approaches, our scheme does not rely on breaking Lorentz reciprocity. Rather, we engineer the interplay between pairs of superconducting transmon qubits and a microwave transmission line, in such a way that two delocalized orthogonal excitations emit (and absorb) photons propagating in opposite directions. We show how such cascaded quantum devices can be exploited to passively probe and measure complex many-body operators on quantum registers of stationary qubits, thus enabling the heralded transfer of quantum states between distant qubits, as well as the generation and manipulation of stabilizer codes for quantum error correction.

npj Quantum Information (2020)6:32; https://doi.org/10.1038/s41534-020-0261-9

\section{INTRODUCTION}

Over the last two decades, superconducting circuit technologies have emerged among the most promising platforms for realizing quantum processors ${ }^{1-4}$. One avenue consists in designing quantum networks in a modular approach, where distant stationary qubits interact by exchanging photons as "flying qubits" propagating in waveguides ${ }^{5}$. As the size of experiments and number of qubits in quantum networks scale in complexity, controllable routing of quantum information between distinct components becomes a requirement ${ }^{6}$. In most current experiments, this task is taken care of using ferrite junction circulators, which break Lorentz reciprocity via the Faraday effect ${ }^{7,8}$. However, as these devices are bulky, lossy, and use large magnetic fields, they are not suitable for on-chip integration, and new, scalable alternatives must be developed. To address this challenge, several approaches were proposed in recent years. Most strategies require active devices $^{9-18}$, where reciprocity is broken by the interplay of several pump fields with precise phase relations, at the cost of adding energy to the system. On the other hand, passive devices have also been proposed based on superconducting junction rings, where circulation is obtained using a constant flux bias; these are however highly sensitive to charge noise ${ }^{19,20}$. In addition, quantum devices building on superconducting qubits strongly coupled to 1D microwave waveguides are being developed, where the reflection and transmission of itinerant photons is externally controlled, including for instance singlephoton routers ${ }^{21}$ and transistors 22,23 , unidirectional phonon transducers ${ }^{24}$, or quantum diodes ${ }^{25,26}$.

In this work, we tackle the problem of quantum information routing from a different angle; rather than circulators or reflectors for itinerant photons, we design effective integrated qubits as composite objects coupled to a meandering 1D transmission line (see Fig. $1 \mathrm{a}-\mathrm{c}$ ), with the requirement that photons propagating in one direction are absorbed and reemitted along the same direction, without breaking reciprocity. Coherently driving several such unidirectional quantum emitters through the transmission line gives rise to an effective cascaded driven-dissipative dynamics, as represented in Fig. 1d, where photons radiated by each emitter coherently drives other emitters downstream; in the literature, this paradigm is sometimes referred to as "chiral quantum optics" 27 , and features interesting steady-state properties, as will be discussed below.

In analogy to "giant" artificial atoms ${ }^{28-34}$, which couple to a photonic or phononic waveguide at several points separated by distances comparable to the wavelength, our approach consists in designing a giant unidirectional emitter (GUE), here realized using two artificial atoms as anharmonic oscillators, as represented in Fig. 1a. These atoms are coupled to a waveguide, at two points separated by a distance $\bar{d} \sim \lambda_{0} / 4$, with $\lambda_{0}$ the photon wavelength. By designing the interaction between artificial atoms, our composite object effectively admits a $V$-level structure with two delocalized excited states $|L\rangle \sim\left(i\left|1_{1} 0_{2}\right\rangle+\left|0_{1} 1_{2}\right\rangle\right) / \sqrt{2}$ and $|R\rangle \sim$ $\left(\left|1_{1} 0_{2}\right\rangle+i\left|0_{1} 1_{2}\right\rangle\right) / \sqrt{2}$ (with $\left|n_{k}\right\rangle$ denoting Fock state $n=0,1, \ldots$ of atom $k=1,2$ ), with the remarkable property that their transitions to the ground state $\left|0_{1} O_{2}\right\rangle$ couple respectively only to left- and right-propagating modes of the waveguide [see Fig. 1b], which is due to a destructive interference in the photon emission (and absorption). Below we will analyze an implementation of this model with superconducting transmon qubits coupled via a superconducting quantum interference device (SQUID) [see Fig. 1c].

As we will show later on, these composite emitters can be used as unidirectional photonic interfaces for additional long-lived stationary qubits (represented below in Fig. 3), which has immediate applications for quantum information processing and quantum computing. In our approach, quantum information is manipulated and directed passively, using an itinerant probe field as "flying qubit" propagating in the waveguide. This forms a naturally scalable architecture for quantum networking, which we will illustrate in particular with the realization of quantum state transfer between distant stationary qubits, and with the generation and manipulation of stabilizer codes for quantum error correction $^{35}$. Our architecture is passive and tunable in situ, and, as we will show, the required experimental parameters and imperfections are achievable with current technology.

\footnotetext{
${ }^{1}$ Center for Quantum Physics, University of Innsbruck, Innsbruck A-6020, Austria. ${ }^{2}$ Institute for Quantum Optics and Quantum Information, Austrian Academy of Sciences, Innsbruck A-6020, Austria. ${ }^{3}$ Univ. Grenoble Alpes, CNRS, LPMMC, 38000 Grenoble, France. ${ }^{4}$ Institute for Experimental Physics, University of Innsbruck, A-6020 Innsbruck, Austria.

巴email: pierre-olivier.guimond@uibk.ac.at
} 
(a)

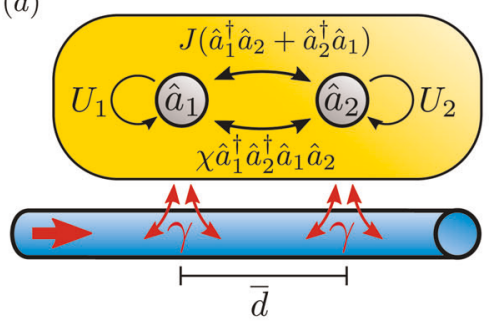

(d)

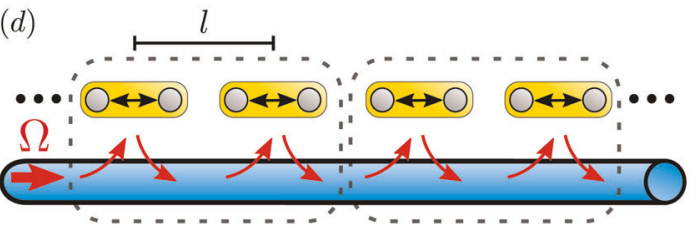

$|D\rangle$ (b)

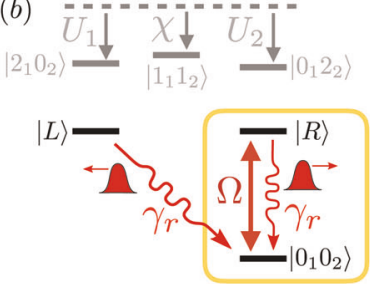

(c)

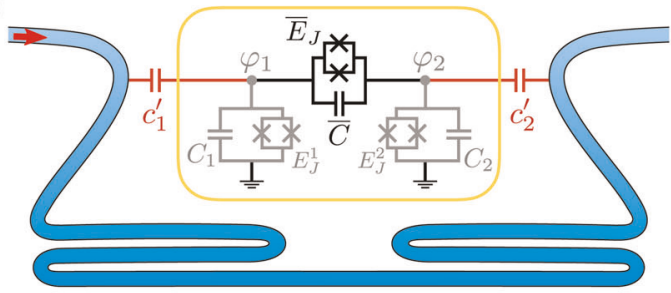

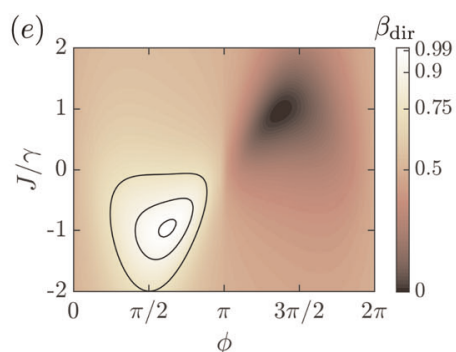

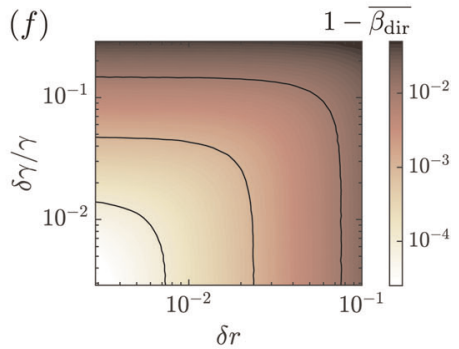

Fig. 1 Unidirectional coupling of quantum emitters to a transmission line. a Model for realizing a giant unidirectional emitter (GUE) using non-linear coupling between two artificial atoms coupled to a waveguide. $\mathbf{b}$ Corresponding level structure obtained with specific parameters (see text). An effective two-level system with states $\left|0_{1} 0_{2}\right\rangle$ and $|R\rangle$ is obtained, which couples to right-propagating modes of the transmission line. c Superconducting circuit implementation, where two transmons $(k=1,2)$ are coupled at two points to a meandering transmission line, and interact via a SQUID. d Driven-dissipative cascaded quantum network realized with several GUEs as effective two-level emitters unidirectionally coupled to a transmission line. The system dissipates towards a pure steady-state with emitters pairing up in an entangled state $|D\rangle$. e Directionality $\beta_{\text {dir }}$ of emitted photons, with $\Delta_{k}=0$ and $r_{k}=0.2, \gamma_{k}=\gamma$. f Averaged directionality $\overline{\beta_{\text {dir }}}$ for $J=J_{\text {opt }}, \phi=\phi_{\mathrm{opt}}$ and $\Delta_{k}=0$, obtained with uniformly distributed $r_{1}, r_{2}, \gamma_{1}$, and $\gamma_{2}$, with means $\overline{r_{k}}=0.2, \overline{\gamma_{k}}=\gamma$ and standard deviations $\sqrt{{\overline{r_{k}^{2}}}^{2} \bar{r}_{k}^{2}}=\delta r_{,} \sqrt{\overline{\gamma_{k}^{2}}-\bar{\gamma}_{k}^{2}}=\delta \gamma$.

\section{RESULTS}

Our results presented below are organized as follows. First we describe and analyse the design of giant unidirectional emitters (GUEs) as composite artificial atoms with an effective V-level structure, with each transition absorbing and emitting photons along a single direction in a waveguide, and present a possible implementation with superconducting transmon qubits. Next, we study the cascaded driven-dissipative dynamics arising when several such unidirectional emitters are driven via the waveguide. Finally, in the last part we describe how these emitters can act as unidirectional photonic interfaces for additional long-lived stationary qubits, which enables applications for quantum networking such as quantum state transfer between distant stationary qubits, and the generation and manipulation of stabilizer codes for quantum error correction.

Model of unidirectional quantum emitters

Our model for designing unidirectional quantum emitters is represented in Fig. 1a, and consists of two interacting artificial atoms as anharmonic oscillators coupled at two distant points to a waveguide. The dynamics of these two atoms, within the rotating wave approximation, is described by the Hamiltonian (with $\hbar=1$ )

$$
\begin{aligned}
\hat{H}_{a}= & \sum_{k=1}^{2} \omega_{k} \hat{a}_{k}^{\dagger} \hat{a}_{k}-\left(U_{k} / 2\right) \hat{a}_{k}^{\dagger} \hat{a}_{k}^{\dagger} \hat{a}_{k} \hat{a}_{k} \\
& +J\left(\hat{a}_{1}^{\dagger} \hat{a}_{2}+\hat{a}_{2}^{\dagger} \hat{a}_{1}\right)-x \hat{a}_{1}^{\dagger} \hat{a}_{1} \hat{a}_{2}^{\dagger} \hat{a}_{2} .
\end{aligned}
$$

Here $\omega_{k}$ is the transition frequency of each atom $k, U_{k}$ denotes their anharmonicity, and $\hat{a}_{k}$ is their annihilation operator, which satisfies $\left[\hat{a}_{k}, \hat{a}_{l}^{\dagger}\right]=\delta_{k, l}$. The second line in Eq. (1) describes the interaction between atoms, with linear exchange interaction rate $J$, and non-linear cross-Kerr frequency $X$, which can be implemented with two superconducting transmon qubits coupled via a SQUID (see Fig. 1c and discussion below).

The waveguide has a continuous spectrum of modes described over the relevant bandwidth by the bare Hamiltonian $\hat{H}_{\mathrm{ph}}=\int d \omega \omega\left[\hat{b}_{R}^{\top}(\omega) \hat{b}_{R}(\omega)+\hat{b}_{L}^{\dagger}(\omega) \hat{b}_{L}(\omega)\right]$, where $\hat{b}_{d}(\omega)$ is the annihilation operator for photons with frequency $\omega$ propagating to the right (with $d=R$ ) or to the left (with $d=L$ ), and satisfies $\left[\hat{b}_{d}(\omega), \hat{b}_{d^{\prime}}\left(\omega^{\prime}\right)\right]=\delta\left(\omega-\omega^{\prime}\right) \delta_{d, d^{\prime}}$. Finally, the coupling between the atoms and the waveguide yields, within the rotating wave approximation, the Hamiltonian

$$
\begin{aligned}
\hat{H}_{\text {int }}= & \frac{1}{\sqrt{2 \pi}} \int d \omega\left[\hat{b}_{R}^{\dagger}(\omega)\left(e^{i \omega \bar{d} / v_{g}} \hat{L}_{1}+\hat{L}_{2}\right)\right. \\
& \left.+\hat{b}_{L}^{\dagger}(\omega)\left(\hat{L}_{1}+e^{i \omega \bar{d} / v_{g}} \hat{L}_{2}\right)+\text { h.c. }\right] .
\end{aligned}
$$

Here $\hat{L}_{1}=\sqrt{\gamma_{1}}\left(\hat{a}_{1}+r_{2} \hat{a}_{2}\right)$ and $\hat{L}_{2}=\sqrt{\gamma_{2}}\left(\hat{a}_{2}+r_{1} \hat{a}_{1}\right)$ are the coupling operators associated to each coupling point, with coupling rates $\gamma_{k}$ (which we assume constant over the relevant bandwidth) and small cross-coupling coefficients $r_{k}$ which are not needed per se for the design of unidirectional emitters, but arise in our proposed implementation as discussed below. We also defined is the distance of separation $\bar{d}$ between the two coupling points along the waveguide, and the group velocity $v_{g}$ of photons in the waveguide.

Within a markovian approximation (i.e., assuming $\gamma_{k} \bar{d} / v_{g} \ll 1$ ), the dynamics of the field can be integrated and treated as a reservoir for the atoms, and we obtain for the Heisenberg equation of motion for an arbitrary atomic operator $\hat{O}(t)$ the quantum Langevin equation (see details in Supplementary Methods I)

$$
\begin{aligned}
\frac{d}{d t} \hat{O}(t)= & -i\left[\hat{O}, \hat{H}_{\mathrm{eff}}\right]+\sum_{d=R, L} \hat{L}_{d}^{\dagger} \hat{O} \hat{L}_{d}-\frac{1}{2}\left\{\hat{L}_{d}^{\dagger} \hat{L}_{d}, \hat{O}\right\} \\
& +\sum_{d=R, L}\left[\hat{b}_{d}^{\text {in }}(t)\right]^{\dagger}\left[\hat{O}, \hat{L}_{d}\right]+\left[\hat{L}_{d}^{\dagger}, \hat{O}\right] \hat{b}_{d}^{\text {in }}(t),
\end{aligned}
$$

expressed in an interaction picture with respect to the waveguide Hamiltonian $\hat{H}_{\text {ph }}$ and in a rotating frame with respect to the photon frequency $\omega_{0}=2 \pi v_{g} / \lambda_{0}$. Here the effective Hamiltonian 
reads

$$
\begin{aligned}
\hat{H}_{\text {eff }}= & -\sum_{k=1}^{2} \Delta_{k} \hat{a}_{k}^{\dagger} \hat{a}_{k}-\left(U_{k} / 2\right) \hat{a}_{k}^{\dagger} \hat{a}_{k}^{\dagger} \hat{a}_{k} \hat{a}_{k}-x \hat{a}_{1}^{\dagger} \hat{a}_{1} \hat{a}_{2}^{\dagger} \hat{a}_{2} \\
& +J\left(\hat{a}_{1}^{\dagger} \hat{a}_{2}+\hat{a}_{2}^{\dagger} \hat{a}_{1}\right)+\sin (\phi)\left(\hat{L}_{2}^{\dagger} \hat{L}_{1}+\hat{L}_{1}^{\dagger} \hat{L}_{2}\right),
\end{aligned}
$$

with $\Delta_{k}=\omega_{0}-\omega_{k}$, where the last term emerges from a coherent exchange of photons propagating in the waveguide between the two coupling points, with $\phi=\omega_{0} \bar{d} / v_{g}$ the phase acquired by a photon in the propagation. On the other hand, the collective coupling operators in Eq. (3) represent the collective couplings of the atoms to right- and left-propagating photons due to interference of photon emission and absorption in the reservoir, and are defined respectively as $\hat{L}_{R}(t)=e^{i \phi} \hat{L}_{1}(t)+\hat{L}_{2}(t)$ and $\hat{L}_{L}(t)=\hat{L}_{1}(t)+e^{i \phi} \hat{L}_{2}(t)$. Finally, $\hat{b}_{d}^{\text {In }}(t)$ represents the input fields of the waveguide propagating along direction $d$, and is related to the output fields via ${ }^{36}$

$\hat{b}_{d}^{\text {out }}(t)=\hat{b}_{d}^{\text {in }}(t)+\hat{L}_{d}(t)$,

with $\left[\hat{b}_{d}^{\text {in/out }}(t),\left(\hat{b}_{d^{\prime}}^{\text {in/out }}\left(t^{\prime}\right)\right)^{\dagger}\right]=\delta\left(t-t^{\prime}\right) \delta_{d, d^{\prime}}$. The emergence of unidirectional coupling between propagating photons and the composite two-atom system, from Eqs. (3) and (5), occurs under the following two conditions.

(I) First, the two collective coupling operators $\hat{L}_{R}$ and $\hat{L}_{L}$ must be orthogonal, i.e., $\left[\hat{L}_{L}^{\dagger}, \hat{L}_{R}\right]=0$, such that each operator $\hat{L}_{d}$ couples only to the corresponding input fields $\hat{b}_{d}^{\text {th }}(t)$ in Eq. (3). Here, this condition requires the system parameters to be symmetric, i.e., $r_{1}=r_{2}=r$ and $\gamma_{1}=\gamma_{2}=\gamma$, while the propagation phase must be set to $\phi=\phi_{\mathrm{opt}}$, with the optimal propagation phase $\phi_{\text {opt }}=\pi / 2+2 \arctan (r)$. With these parameters, the collective coupling operators reduce to $\hat{L}_{R / L}=\sqrt{Y_{r}} \hat{a}_{R / L}$, up to an irrelevant phase factor, with the definition of two orthogonal delocalized atomic modes $\hat{a}_{R}=$ $\left(i \hat{a}_{1}+\hat{a}_{2}\right) / \sqrt{2}$ and $\hat{a}_{L}=\left(\hat{a}_{1}+i \hat{a}_{2}\right) / \sqrt{2}$, and where the effective coupling strength of the system to the waveguide is given by $\gamma_{r}=2 \gamma\left(1+2 r \cos \left[\phi_{\text {opt }}\right]+r^{2}\right)$. We note that the cross-coupling coefficients $r_{k}$ are not necessary ingredients in our model of unidirectional emitters, and in the simpler case where $r \approx 0$ we obtain $\phi_{\text {opt }} \approx \pi / 2$, yielding $\gamma_{r} \approx 2 \gamma$.

(II) Second, the excitations associated to these two modes $\hat{a}_{R}$ and $\hat{a}_{L}$ must be eigenstates of the effective Hamiltonian $\hat{H}_{\text {eff }}$. For states with a single atomic excitation, i.e., $|R\rangle=\hat{a}_{R}^{\dagger}|G\rangle$ and $|L\rangle=\hat{a}_{L}|G\rangle$ with $|G\rangle=\left|0_{1} 0_{2}\right\rangle$ the ground state of both atoms, this is achieved by taking symmetric detunings $\Delta_{1}=$ $\Delta_{2} \equiv \Delta+2 r y \sin \left(\phi_{\text {opt }}\right)$ and $J=J_{\text {opt }}$ with the optimal hopping rate given by $J_{\text {opt }}=-\gamma\left(1+r^{2}\right) \sin \left(\phi_{\text {opt }}\right)$. In the regime $r \approx 0$, this condition can be understood as the requirement for the direct hopping (with rate $J$ ) to exactly cancel the contribution from waveguide-mediated photon exchanges (with rate $\gamma \sin (\phi)$ ) in Eq. (4), as these terms couple the two excited states $|R\rangle$ and $|L\rangle$. If this condition is satisfied, these two states then become eigenstates of $\hat{H}_{\text {eff }}$ with eigenenergies $-\Delta$. The non-linear cross-Kerr interaction with frequency $X$, on the other hand, is introduced in the model in order to prevent the excitation of the doubly-excited state $\left|1_{1} 1_{2}\right\rangle$ when driving the system via the input fields, as we will consider below.

When these two conditions are fulfilled, the composite emitter will absorb and reemit propagating photons along the same direction. In order to assess this directionality in a more general case, we assume the emitter is prepared in state $|R\rangle$ at time $t=0$ with the waveguide in the vacuum state, and solve the dynamics of the system, which yields the emission of a photon in the waveguide, with the emitter returning to its ground state $|G\rangle$. The temporal shapes of the wavepacket amplitudes of the emitted photon propagating to the right/left are then obtained using a Wigner-Weisskopf ansatz (see details in Supplementary Methods I) as $f_{R / L}(t) \equiv\left\langle G\left|\hat{b}_{R / L}^{\text {out }}(t)\right| R\right\rangle=\langle G| \hat{L}_{R / L} \mathcal{L}^{-1}\left[\hat{F}^{-1}(s)|R\rangle\right]$, where $\mathcal{L}[\cdot](s)$ denotes the Laplace transform, and the evolution of the atomic excitation amplitudes is governed by the operator

$\hat{F}(s)=s+i \hat{H}_{\text {eff }}+\frac{1}{2}\left(\hat{L}_{R}^{\dagger} \hat{L}_{R}+\hat{L}_{L}^{\dagger} \hat{L}_{L}\right)$.

We then define the directionality of photon emission as $\beta_{\text {dir }}=\int_{0}^{\infty}\left|f_{R}(t)\right|^{2} d t$. This directionality of emitted photons is represented in Fig. 1e, f. Figure 1e shows that very good directionalities can be achieved even with relatively large imprecisions on $J$ and $\phi$ around their optimal values, e.g. due to fabrication imperfections. Here we obtain $\beta_{\text {dir }}>99 \%$ for $\left|J-J_{\text {opt }}\right|$ $\lesssim \gamma / 10$ and $\left|\phi-\phi_{\text {opt }}\right| \lesssim \pi / 10$. This robustness to imperfections is also observable in Fig. 1f, where we show the average directionality $\overline{\beta_{\text {dir }}}$ obtained with random static deviations of $r_{k}$ and $\gamma_{k}$. We obtain $\overline{\beta_{\text {dir }}}>99 \%$ as long as the fluctuation in the coupling parameters are below $\delta \gamma \lesssim 0.1 \gamma$ and $\delta r \lesssim 0.05$.

Implementation with superconducting circuits

Our model can be implemented with the circuit represented in Fig. 1c, which consists of two superconducting transmon qubits $(k=1,2)$ with flux-tunable Josephson energies $E_{j}^{k}$ and charging energies $E_{C}^{k}=e^{2} /\left(2 C_{k}^{\text {eff }}\right)^{37}$, where $e$ is the elementary charge and $C_{k}^{\text {eff }}$ are the effective transmon capacitances (see details in Methods). The interaction between transmons is mediated by a SQUID, acting as a non-linear element with flux-tunable Josephson energy $\bar{E}_{J}$ and with capacitance $\bar{C}$. We note that such tunable nonlinear couplings mediated by Josephson junctions were demonstrated in recent experiments ${ }^{38-40}$, and find applications for quantum simulation ${ }^{41-43}$ and quantum information processing ${ }^{22}$.

Following standard quantization procedures, the Hamiltonian for the circuit can be expressed as in Eq. (1) (see details in Methods). In particular, analytical insight on the resulting system parameters can be gained in the regime of weakly coupled transmons, with $E_{C}^{k} \ll E_{J}^{k}, \bar{E}_{J} \ll E_{J}^{k}$ and $\bar{C} \ll C_{k}$. In this limit, an estimation of the various parameters of the model can be made in terms of the circuit parameters, with the atomic transition frequencies taking the expression $\omega_{k} \approx \sqrt{8 E_{J}^{k}} E_{C}^{k}$, while the atomic anharmonicities read $U_{k} \approx E_{C}^{k}$. The interaction between atoms contains a linear hopping term $J=J_{C}-J_{l}$, with a capacitive $\left(J_{C}\right)$ and an inductive $\left(J_{l}\right)$ contribution reading

$$
J_{C} \approx \omega_{0} \frac{\bar{C}}{2 \sqrt{C_{1}^{\text {eff }} C_{2}^{\text {eff }}}}, J_{l} \approx \omega_{0} \frac{\bar{E}_{J}}{2 \sqrt{E_{J}^{1} E_{J}^{2}}},
$$

while the cross-Kerr interaction term reads

$$
x=2 \bar{E}_{J} \sqrt{\frac{E_{C}^{1} E_{C}^{2}}{E_{J}^{1} E_{J}^{2}}} .
$$

We note that the three Josephson energies in Fig. 1c can be independently controlled via flux biases, allowing for an independent in situ fine-tuning of the detunings $\Delta_{k}=\omega_{0}-\omega_{k}$ and the hopping rate $J$. The couplings to the waveguide on the other hand are given by $\gamma_{k}=\left(c_{k}^{\prime} / C_{k}^{\text {eff }}\right)^{2} \omega_{0} e^{2} Z_{0} \sqrt{E_{J}^{k} /\left(8 E_{C}^{k}\right)}$, with $c_{k}^{\prime}$ the coupling capacitances and $Z_{0}$ the transmission line impedance ${ }^{44,45}$. The capacitance $\bar{C}$ introduces as well small crosscoupling coefficients $r_{k}=\bar{C} / C_{k}^{\text {eff }}$, resulting in photon emission from each artificial atom via both coupling points.

Driven-dissipative dynamics of cascaded quantum networks Although the properties of unidirectional emission of our GUE studied above preserve Lorentz reciprocity, i.e., they are invariant under the exchange of left- and right-propagating modes, driving 
the system through the waveguide allows one to effectively achieve non-reciprocal interactions between artificial atoms. A paradigmatic example of such a situation is represented in Fig. 1d, where several GUEs are coherently driven via right-propagating modes, thus driving the $\hat{a}_{R}$ transition as represented in Fig. $1 \mathrm{~b}$. Photons emitted by each emitter will then also propagate to the right, leading to an effective cascaded quantum dynamics, where each GUE drives the other ones downstream, without any backaction $^{46-48}$.

This scenario has been studied in recent years in a different context, in a field known in the literature as "chiral quantum optics"27, which originated from experiments with quantum emitters in the optical domain, such as atoms ${ }^{49-52}$ or quantum $\operatorname{dots}^{53-56}$, coupled to photonic 1D nanostructures. The strong confinement of light in these structures gives rise to a so-called "spin-momentum locking" effect ${ }^{57}$, allowing for unidirectional couplings between photons and emitters which, in an analogous way to our GUE, does not by itself break Lorentz reciprocity. Besides, building on non-local couplings of quantum emitters to 1D reservoirs, chiral quantum optical systems could also be realized in AMO platforms with broken reciprocity ${ }^{58-60}$. While photon losses inherent to optical platforms form experimental challenges, the near-ideal mode matching of artificial atoms coupled to 1D transmission lines presents new opportunities to realize this paradigm, in the microwave domain ${ }^{21,61}$. Interestingly, it has been predicted that, for several quantum emitters, the ensuing cascaded dynamics in the presence of a coherent drive results in the dissipative preparation of quantum dimers, with quantum emitters pairing up in a dark, entangled state ${ }^{62-64}$, as we will show below.

In order to study the dynamics of an ensemble of $N$ GUEs (labeled $n=1, \ldots, N$ ) interacting via a common waveguide, we employ the SLH input-output formalism ${ }^{65-67}$. The SLH framework provides a methodical approach for modeling such composite quantum systems interacting via the exchange of propagating photons, where we assume that non-Markovian effects, due e.g. to the finite propagation time of photons exchanged by the emitters ${ }^{68}$, can be neglected. As detailed in the Supplementary Methods IV, the dynamics of the network of $N$ GUEs can then be obtained from the input-output properties of each individual GUE, by recursively applying composition rules of the SLH formalism in a "bottom-up" fashion. The evolution of an arbitrary atomic operator $\hat{O}(t)$ in the rotating frame then obeys a quantum Langevin equation as expressed in Eq. (3), with a redefinition of the effective Hamiltonian and of the coupling operators. Denoting the various parameters and operators associated with each GUE with a corresponding superscript $n$, we obtain for the effective Hamiltonian

$$
\begin{aligned}
\hat{H}_{\text {eff }}^{\text {tot }}= & \sum_{n} \hat{H}_{\text {eff }}^{n}-\frac{i}{2} \sum_{n, m<n}\left[\left(\hat{L}_{R}^{n}\right)^{\dagger} \hat{L}_{R}^{m} e^{i \tilde{\phi}(n-m)}-\text { h.c. }\right] \\
& -\frac{i}{2} \sum_{n, m>n}\left[\left(\hat{L}_{L}^{n}\right)^{\dagger} \hat{L}_{L}^{m} e^{i \tilde{\phi}(m-n)}-\text { h.c. }\right],
\end{aligned}
$$

with the photon propagation phase $\tilde{\phi}=\omega_{0} l / v_{g}$ where $I$ is the distance between two neighbouring composite emitters along the waveguide. We note that the two new terms in Eq. (9) correspond to excitation exchange interactions between different GUEs, mediated respectively by right- and left-propagating photons. For the coupling operators on the other hand, we obtain $\hat{L}_{R}^{\text {tot }}=$ $\sum_{n} e^{i \tilde{\phi}(N-n)} \hat{L}_{R}^{n}$ and $\hat{L}_{L}^{\text {tot }}=\sum_{n} e^{i \tilde{\phi}(n-1)} \hat{L}_{L}^{n}$, which represent interference in the atom-field coupling between the emitters.

The presence of a coherent drive via the right-propagating waveguide modes, with amplitude $a(t)$ [and corresponding Rabi frequency $\Omega(t)=\sqrt{Y_{r}} a(t)$ ], can be accounted for by assuming the initial state of the waveguide $\left|a_{R}\right\rangle$ satisfies $\hat{b}_{d}^{\text {In }}(t)\left|a_{R}\right\rangle=a(t) \delta_{d, R}\left|a_{R}\right\rangle$. Writing $\langle\hat{O}(t)\rangle=\operatorname{Tr}[\hat{O} \rho(t)]$, with $\hat{\rho}$ the atomic density matrix, the temporal evolution from Eq. (3) then yields the master equation

$$
\begin{aligned}
\frac{d}{d t} \hat{\rho}= & -i\left[\hat{H}_{\text {eff }}^{\text {tot }}-i a(t)\left(\hat{L}_{R}^{\text {tot }}\right)^{\dagger}+i a^{*}(t) \hat{L}_{R}^{\text {tot }}, \hat{\rho}\right] \\
& +\mathcal{D}\left[\hat{L}_{R}^{\text {tot }}\right] \hat{\rho}+\mathcal{D}\left[\hat{L}_{L}^{\text {tot }}\right] \hat{\rho},
\end{aligned}
$$

where $\mathcal{D}[\hat{a}] \hat{\rho}=\hat{a} \hat{\rho} \hat{a}^{\dagger}-\frac{1}{2}\left\{\hat{a}^{\dagger} \hat{a}, \hat{\rho}\right\}$. Equation (10) allows to access the evolution and steady-state values of observables with a finite drive amplitude $a$. In order to account for additional imperfections, we also add in Eq. (10) dephasing terms $2 \gamma_{\varphi} \sum_{n, k} \mathcal{D}\left[\left(\hat{a}_{k}^{n}\right)^{\dagger} \hat{a}_{k}^{n}\right]$ and non-radiative decay terms $\gamma_{\mathrm{nr}} \sum_{n, k} \mathcal{D}\left[\hat{a}_{k}^{n}\right]$.

In Fig. 2a, b we represent the ratio of left- and right-propagating emitted photons obtained in the steady-state of the dynamics for $N=1$, with $J$ and $\phi$ set to their optimal values, and a constant real Rabi frequency $\Omega$ (i.e., the drive frequency is $\omega_{0}$ ). Figure 2a shows that, since directionality arises in our setup as interference of emission of the two atoms, the dephasing rate $\gamma_{\varphi}$ spoils the interference and induces some emission to the left with an intensity scaling linearly for low Rabi frequency $\Omega$. As $\Omega$ increases with respect to the effective anharmonicities $X$ and $U_{k}$ the intensity of left-propagating photons increases, as states with more than a single excitation get populated. For instance, the state $\left|1_{1} 1_{2}\right\rangle$ in Fig. $1 \mathrm{~b}$ can be excited by absorbing two (rightpropagating) photons from the drive, and can afterwards decay towards state $|L\rangle$ by emitting a photon propagating to the left. This increase in the population of multiple-excitation states is also observed as the dashed red curves in Fig. $2 \mathrm{~b}$, and we thus require $\Omega \ll X$ in order to retain a two-level dynamics. We also note that when $X=U_{1}=U_{2}$ in Fig. $2 \mathrm{~b}$, the emission to the left vanishes even when states with several excitations are populated, as for these parameters states with several excitations $\left(\hat{a}_{R}^{\dagger}\right)^{n_{R}}\left(\hat{a}_{L}^{\dagger}\right)^{n_{L}}|G\rangle$ become eigenstates of $\hat{H}_{\text {eff }}^{\text {tot }}$ for all $n_{R / L} \geq 0$, thus preserving the property of unidirectional emission. Note that in the regime of weakly coupled transmons $\left(\bar{C} \ll C_{k}\right.$ and $\left.\bar{E}_{J} \ll E_{\jmath}^{k}\right)$ considered above, the value of $U$ is limited by the fact that, from Eq. (8) and $U_{k} \approx E_{C}^{k}$, we have $x \ll 2 \sqrt{U_{1} U_{2}}$. Achieving larger values thus requires going beyond the weak coupling regime. This is discussed in the Supplementary Methods II, where we also study the validity of the analytical expressions for the effective model in Eqs. (7) and (8). Typical achievable values for $X$ range from 0 to $\sim 2 \pi \times 50 \mathrm{MHz}$ with $U_{k}=$ $2 \pi \times 300 \mathrm{MHz}$.

In the ideal case where the parameters satisfy the properties of unidirectional coupling and the anharmonicities $X$ and $U_{k}$ are large enough with respect to the Rabi frequency $\Omega$ of the drive, the state of the emitters will thus remain within the two-level manifold $\otimes_{n}\left\{|G\rangle_{n},|R\rangle_{n}\right\}$. Denoting here $\hat{\sigma}_{+}^{n}=e^{i \tilde{\phi} n}|R\rangle_{n}\langle G|$, the dynamics of Eq. (10) then reduces to a cascaded master equation ${ }^{46,47}$

$\frac{d}{d t} \hat{\rho}_{\text {eff }}=-i \hat{H}_{\text {nh }} \hat{\rho}_{\text {eff }}+i \hat{\rho}_{\text {eff }} \hat{H}_{\text {nh }}^{\dagger}+\hat{L}_{R}^{\text {tot }} \hat{\rho}_{\text {eff }}\left(\hat{L}_{R}^{\text {tot }}\right)^{\dagger}$,

where $\hat{\rho}_{\text {eff }}$ denotes the density matrix of the system expressed in the reduced $2^{N}$-dimensional manifold, and where the effective non-Hermitian Hamiltonian reads, assuming $\Omega$ real,

$$
\begin{aligned}
\hat{H}_{\mathrm{nh}}= & -\Delta \sum_{n} \hat{\sigma}_{+}^{n} \hat{\sigma}_{-}^{n}-i \Omega\left(\hat{\sigma}_{+}^{n}-\hat{\sigma}_{-}^{n}\right) \\
& -i \frac{\gamma_{r}}{2} \sum_{n} \hat{\sigma}_{+}^{n} \sigma_{-}^{n}-i \gamma_{r} \sum_{n>m} \hat{\sigma}_{+}^{n} \hat{\sigma}_{-}^{m} .
\end{aligned}
$$

Equation (11) represents the ideal scenario where the coupling between propagating photons and the array of N GUEs is purely unidirectional, which assumes that the directionality parameter satisfies $N\left(1-\beta_{\text {dir }}\right) \ll 1$. The dynamics generated by Eq. (11) then induces an effective non-reciprocal interaction between the qubits: as seen from the expression of Eq. (12), an excitation in each qubit $m$ can be coherently transferred only to qubits $n>m$ 

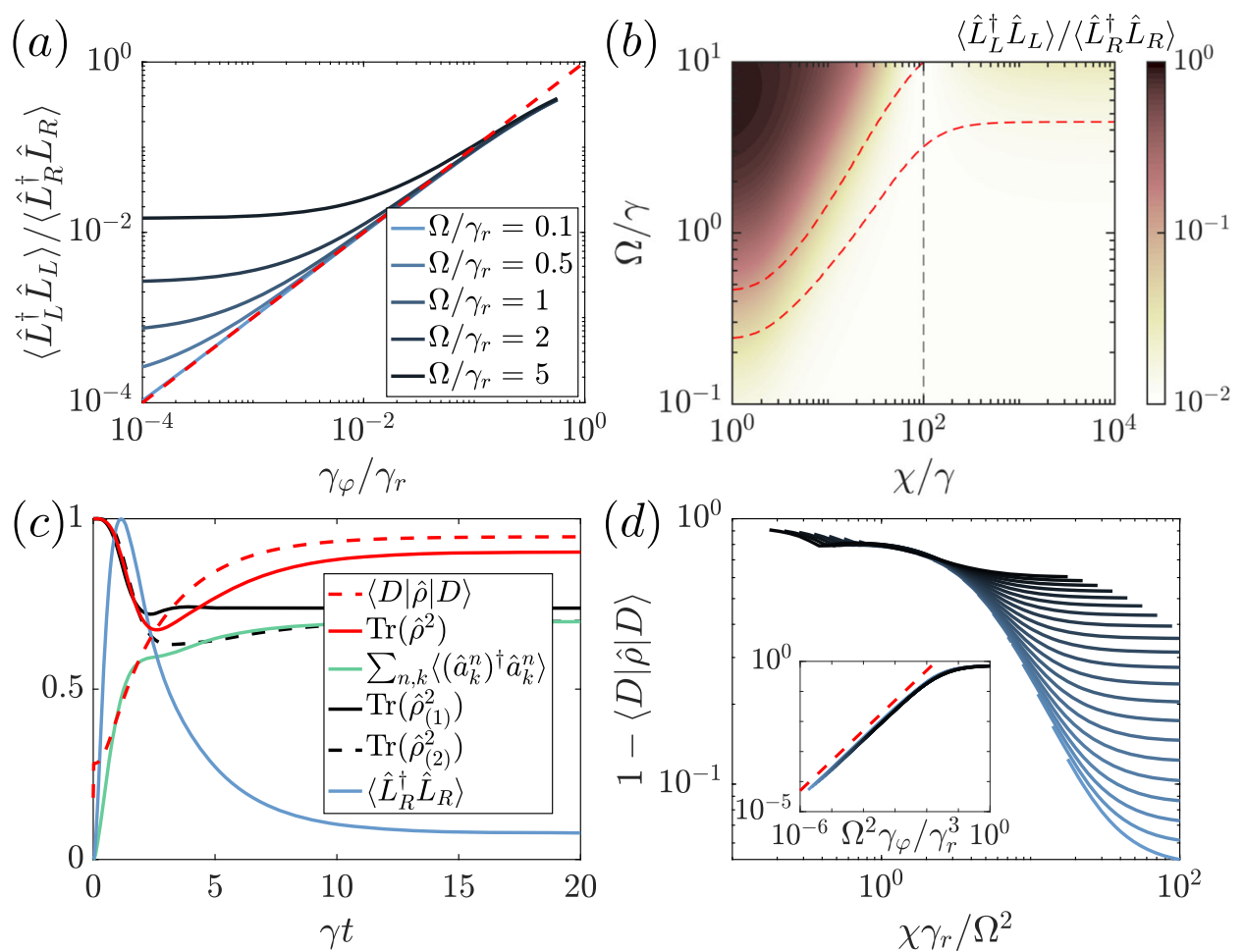

Fig. 2 Driven-dissipative dynamics. a, $\mathbf{b}$ Ratio of left- and right-propagating photon intensities, in the steady-state, emitted by the artificial atoms when coherently driven through the waveguide, with $\gamma_{k}=\gamma_{1} \Delta_{k}=0, r_{k}=0.2, U_{k}=100 \gamma, \gamma_{\mathrm{nr}}=0.01 \gamma, J=J_{\mathrm{opt}}, \phi=\phi_{\mathrm{opt}}$. a $X=50 \gamma$. Dashed red: $\gamma_{\varphi} / \gamma_{r}$. b $\gamma_{\varphi}=0.01 \gamma$. Dashed red: probability of $10^{-2}$ and $10^{-3}$ (resp. top and bottom) of having two or more excitations in the atoms. Dashed gray: $X=U_{1}=U_{2}$. c, d Cascaded dynamics with $N=2$ GUEs, with $r_{k}=0.2, \Delta=0, U_{k}=500 \gamma, \tilde{\phi}=0$, and $\gamma_{\mathrm{nr}}=\gamma_{\varphi}$. c $\Omega=\gamma, X=50 \gamma, \gamma_{\varphi}=$ $0.01 \gamma$. d Steady-state overlap $\langle D|\hat{\rho}| D\rangle$, with $\Omega \in[1,10] \gamma$ (light to dark blue), and $\gamma_{\varphi}=0.01 \gamma$. Inset: $\chi \rightarrow \infty$, red dashed curve $\propto \Omega^{2} \gamma_{\varphi} / \gamma_{r}^{3}$.

located to its right. While the reduced density matrix of any single GUE is in general mixed, for even $N$ and $\Delta=0$ the state of the whole system dissipates towards a pure steady-state $|\Psi\rangle=\bigotimes_{n=1}^{N / 2}|D\rangle_{2 n-1,2 n}$, where, as represented in Fig. 1d, neighbouring qubits pair up as dimers in a two-qubit entangled state ${ }^{62-64}$

$|D\rangle_{2 n-1,2 n} \propto|G\rangle_{2 n-1}|G\rangle_{2 n}-2 \sqrt{2} \frac{\Omega}{\gamma_{r}}|S\rangle_{2 n-1,2 n}$,

with $|S\rangle_{2 n-1,2 n}=\left(|R\rangle_{2 n-1}|G\rangle_{2 n}-|G\rangle_{2 n-1}|R\rangle_{2 n}\right) / \sqrt{2}$. Remarkably, once the system has reached this dark state $|D\rangle$, all photons emitted by qubit $2 n-1$ are coherently absorbed by qubit $2 n$, such that each dimer effectively decouples from the waveguide radiation field.

The dynamics obtained for a pair of $N=2$ GUEs, obtained from Eq. (10), is represented in Fig. 2c, d. In Fig. 2c we observe the purification process described above where, in the steady-state, the system dissipates towards the pure state $|D\rangle$, as represented in the red curves. Strikingly, although the atoms are excited (see green curve), the amount of scattered photons, represented in blue, vanishes in the steady-state, i.e., the system becomes dark and decouples from the waveguide. We note that in the transient dynamics, i.e., before reaching the steady-state, photons are scattered unidirectionally by the emitters, which leads to a decrease of the purity $\operatorname{Tr}\left(\hat{\rho}^{2}\right)$. Moreover, the purity of the reduced density matrix $\hat{\rho}_{(n)}$ for each GUE $n$ remains low in the steady-state (see black curves), as they become entangled. The steady-state overlap $\langle D|\hat{\rho}| D\rangle$ is represented in Fig. 2d, which shows a requirement for a large $X$ with respect to the drive intensity $|\Omega|^{2} / \gamma_{r}$. The effect of imperfections due to dephasing and finite excitation lifetimes is represented in the inset, which shows that the steady-state overlap with the dark state becomes unity in the limit $\chi \rightarrow \infty$ and $\gamma_{\mathrm{nr}}=\gamma_{\phi}=0$.
Quantum information routing for quantum networking and computing

Our approach enables the realization of large scale quantum processing units, where quantum information is processed in local nodes, and routed using unidirectional emitters. The setup we have in mind is represented in Fig. 3a, where we represent a possible such architecture, with a set of stationary atomic qubits acting as quantum register, and GUEs acting as an interface between a waveguide and the stationary qubits. The idea is to mediate effective long-range multi-qubit interactions by using (i) sequences of scattering events induced by unidirectional couplings between a single photon as "flying qubit" and each stationary qubit, (ii) local single-qubit operations, and (iii) linear optics represented by unitary operations $\mathcal{U}_{n}$ acting on two waveguides, including in particular 50/50 beam-splitter operations. The applicability of this architecture is illustrated below for quantum state transfer between distant stationary qubits, as well as the generation and manipulation of stabilizer codes.

The scattering events are designed as follows (see Fig. 3b). Denoting the parameters and operators associated with node $n=$ $1, \ldots N$ with an index $n$, each GUE is initially prepared in its ground state $|G\rangle_{n}$, and returns to this state after the photon scattering. The coupling between each stationary qubit (with states $\left.\left\{|0\rangle_{q, n},|1\rangle_{q, n}\right\}\right)$ and its GUE consists of a purely non-linear crossKerr interaction, which can be described by the Hamiltonian (see details in Supplementary Methods III)

$\hat{H}_{V}^{n}=-|1\rangle_{q, n}\langle 1|\left[V_{1}^{n}\left(\hat{a}_{1}^{n}\right)^{\dagger} \hat{a}_{1}^{n}+V_{2}^{n}\left(\hat{a}_{2}^{n}\right)^{\dagger} \hat{a}_{2}^{n}\right]$,

ideally with identical frequencies $V_{1}^{n}=V_{2}^{n} \equiv V$. The effect of this interaction is then to shift the frequency of the excited states of the GUEs by $V$, conditional on qubit atom $n$ being in state $|1\rangle_{q, n^{\prime}}$ without breaking the properties of unidirectional coupling discussed above. A possible implementation of this interaction 

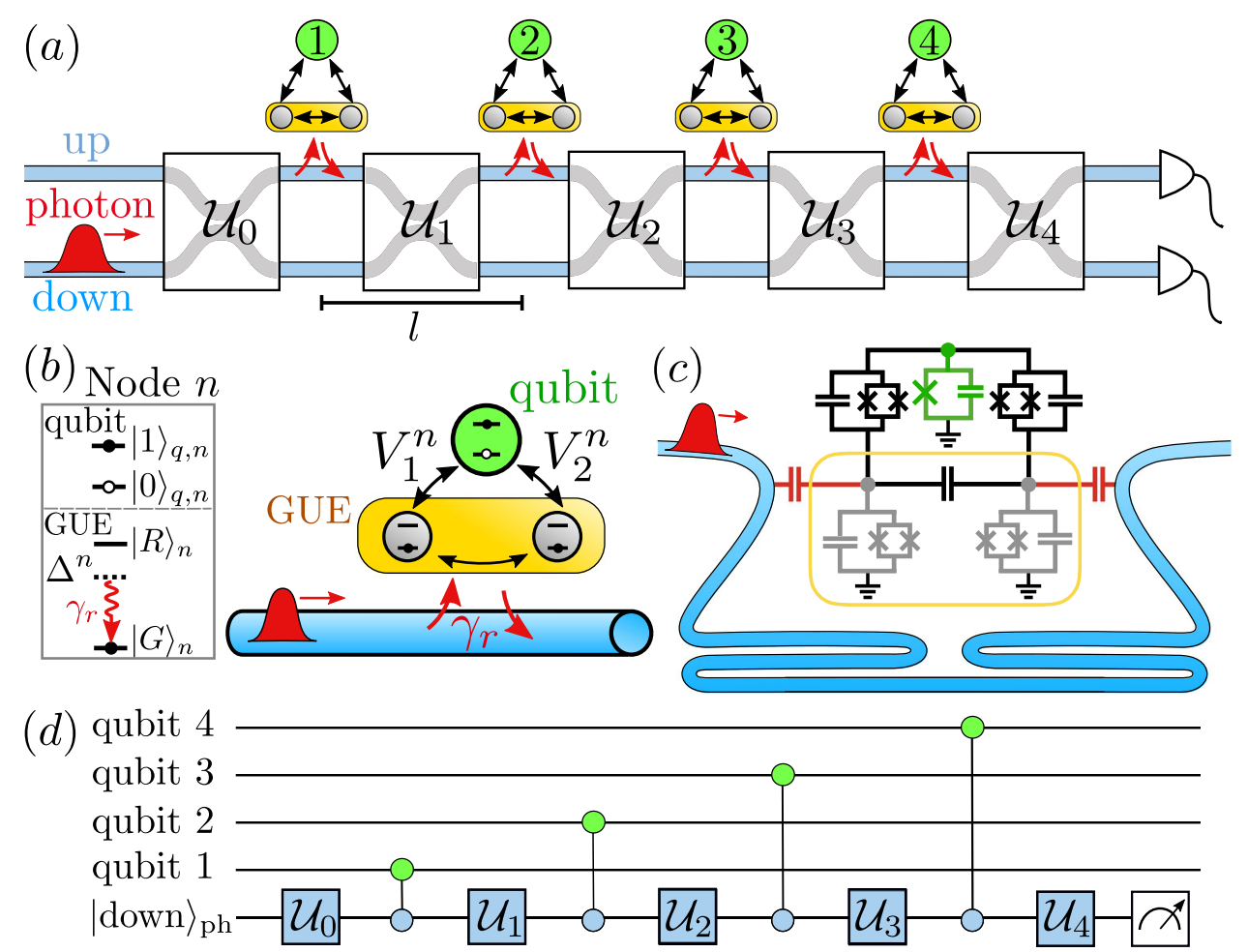

Fig. 3 Architecture for quantum information routing. a An array of qubits $(n=1, \ldots, N)$ is coupled to one of two transmission lines, labeled "up" and "down", via GUEs. A propagating photon scatters sequentially on the qubits, while linear optical elements performing unitary transformations $\mathcal{U}_{n}$ couple the transmission lines. A projective measurement of the qubits is performed upon detecting the photon at the output. b Model for the qubit-GUE interaction in each node $n$ with cross-Kerr frequencies $V_{1}^{n}$ and $V_{2}^{n}$, and c corresponding superconducting implementation adapted from Fig. 1c. $\mathbf{d}$ Quantum circuit realized using the setup in (a), where the double circles represent controlled-Z gates between the qubits and the photon as virtual "flying qubit" with states $|u p\rangle_{p h}$ and $\mid$ down $\rangle_{p h}$, corresponding to the photon propagating in transmission line "up" and "down", respectively.

term with superconducting circuits, adapted from Fig. 1c, is represented in Fig. 3c, where the qubit atom is coupled via two SQUIDs to the GUE atoms. We note that (i) the anharmonicity of the GUEs is inconsequential for the applications considered in this section as we consider the scattering of single photons, hence for simplicity the coupling between the artificial atoms of the GUEs are taken purely capacitive, and (ii) the presence of capacitances in the coupling SQUIDs between the stationary qubit and the GUE induces a small direct coupling between the qubit and the waveguide modes, which could deteriorate the qubit lifetime; however, this coupling can be cancelled by subradiance due to interference in the photon emission from both coupling points, by taking the qubit transition frequency $\omega_{q}$ such that $\omega_{q} \bar{d} / v_{g}$ is an odd multiple of $\pi$ (see details in Supplementary Methods III).

The scattering of a photon on a single node $n$, represented in Fig. $3 b$, is described within the input-output formalism by a singlephoton scattering operator

$\hat{\mathcal{S}}_{d^{\prime}, d}^{n}\left(\nu_{p}, \delta_{p}\right)=\left\langle\operatorname{vac}, \mathrm{G}_{\mathrm{n}}\left|\hat{b}_{d^{\prime}}^{\text {out }}\left(\nu_{p}\right)\left[\hat{b}_{d}^{\text {in }}\left(\delta_{p}\right)\right]^{\dagger}\right| \operatorname{vac}, \mathrm{G}_{\mathrm{n}}\right\rangle$,

where $\mid$ vac,$\left.G_{n}\right\rangle$ denotes the vacuum state of the waveguide, with the GUE in its ground state $|G\rangle_{n^{\prime}}$ and the input and output field operators in the frequency domain are defined via $\hat{b}_{d}^{\text {in/out }}\left(\delta_{p}\right)=(-i / \sqrt{2 \pi}) \int d t \hat{b}_{d}^{\text {in/out }}(t) e^{i \delta_{p} t}$. The single-photon scattering operator represents the action of the temporal evolution operator on qubit $n$, conditional on having an input photon with detuning $\delta_{p}$ (with respect to $\omega_{0}$ ), propagating in direction $d$ [either right $(\mathrm{R})$ or left $(\mathrm{L})]$ be scattered in direction $d^{\prime}$ with detuning $v_{p}$. We consider a right-propagating input photon with frequency distribution given by some function $f\left(\delta_{p}\right)$ with qubit atom $n$ in some state $|\psi\rangle_{q, n}$ and write the state of the system before the scattering as $\mid$ in $\rangle=\int d \delta_{p} f\left(\delta_{p}\right)\left[\hat{b}_{R}^{\text {in }}\left(\delta_{p}\right)\right]^{\dagger} \mid$ vac, $\left.G_{n}\right\rangle|\psi\rangle_{q, n}$. The state after the scattering can then be expressed from Eq. (15) as $\mid$ out $\rangle=\sum_{d^{\prime}} \int d \delta_{p} d \nu_{p} f\left(\delta_{p}\right) \hat{\mathcal{S}}_{d^{\prime}, R}^{n}\left(\nu_{p}, \delta_{p}\right)\left[\hat{b}_{d^{\prime}}^{\text {out }}\left(\nu_{p}\right)\right]^{\dagger} \mid$ vac,$\left.G_{n}\right\rangle|\psi\rangle_{q, n}$.

The single-photon scattering operator in Eq. (15) can be obtained by using the quantum Langevin equation (3) and the input-output relation (5) (see details in Supplementary Methods IV). In particular, under the conditions for unidirectional coupling of the GUEs to the waveguide as discussed above, we find $\hat{\mathcal{S}}_{L, R}^{n}\left(\nu_{p}, \delta_{p}\right)=0$ and $\hat{\mathcal{S}}_{R, R}^{n}\left(\nu_{p}, \delta_{p}\right)=\delta\left(\nu_{p}-\delta_{p}\right) \hat{\sigma}^{n}\left(\delta_{p}\right)$, with the Dirac $\delta$-function representing the conservation of the photon frequency in the scattering, and where

$$
\hat{\sigma}^{n}\left(\delta_{p}\right)=t\left(\Delta^{n}+\delta_{p}\right)|0\rangle_{q, n}\left\langle 0\left|+t\left(\Delta^{n}+\delta_{p}+V\right)\right| 1\right\rangle_{q, n}\langle 1|
$$

with the phase shift $t\left(\delta_{p}\right)=\left(2 i \delta_{p}+\gamma_{r}\right) /\left(2 i \delta_{p}-\gamma_{r}\right)$. The operator $\hat{\sigma}^{n}\left(\delta_{p}\right)$ realizes a generic phase gate on qubit $n$. Assuming the photon has a sharp frequency distribution $f\left(\delta_{p}\right)$ around $\delta_{p}=0$ relative to $\gamma_{r}$, by taking $V=\gamma_{r}$ this phase gate can be parametrized by the value of the tunable detuning $\Delta^{n}$ from GUE $n$. When $\Delta^{n}=$ $-\gamma_{r} / 2$, the two terms in Eq. (16) acquire an opposite $\pi / 2$ phase, and the phase gate becomes the Pauli operator $\hat{\sigma}_{z}^{n}=|0\rangle_{q, n}\langle 0|-| 1\rangle_{q, n}\langle 1|$, up to an irrelevant global phase which can be absorbed in a redefinition of the phase of the output field operator $\hat{b}_{R}^{\text {out }}\left(\delta_{p}\right)$. When $\Delta^{n} \gg \gamma_{r}$ on the other hand, these two terms become identical, and the phase gate reduces to the identity operator $\mathbb{1}$.

This effective unidirectional photon - qubit interaction finds immediate applications for the detection of individual itinerant microwave photons, which is a current technological challenge $\mathrm{e}^{6,69-79}$. This can be realized here with a Ramsey sequence, by preparing the atomic qubit in state $|+\rangle_{q, n^{\prime}}$ with 


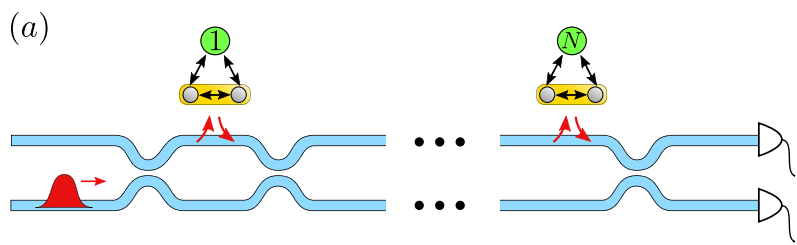

(b)

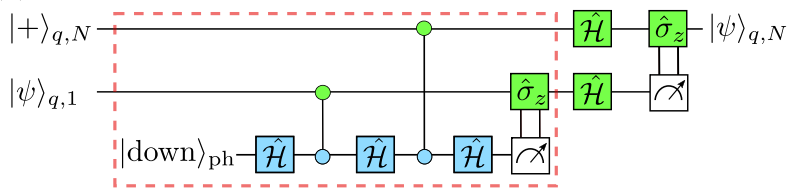

Fig. 4 Protocol for quantum state transfer. a Setup and b corresponding quantum circuit realizing quantum state transfer from qubit atoms 1 to $N$. Hadamard photonic gates $\hat{\mathcal{H}}$ are realized as $50 / 50$ beam-splitters. The dashed red frame represents the action of the scattered photon, with the corresponding quantum circuit realizing a controlled-Z gate between the two qubits. Upon detection of the photon at the output and reading out the final state of qubit 1, the initial superposition state $|\psi\rangle_{q, 1}$ is transferred to $|\psi\rangle_{q, N}$.

$| \pm\rangle \equiv( \pm|0\rangle+|1\rangle) / \sqrt{2}$. With $\Delta^{n}=-\gamma_{r} / 2$, a resonant photon will be scattered unidirectionally by the GUE, while qubit atom $n$ will be left in state $|-\rangle_{a, n}$. The photon can then be detected by measuring the qubit state after applying a Ramsey $\pi / 2$-pulse, which realizes a quantum non-demolition measurement of the itinerant photon, in analogy to the cavity-QED experiments in refs. ${ }^{69-73}$. The resonance frequency $\omega_{0}$ of this detector can be tuned, while the detection bandwidth is given by $\gamma_{r}$ (see details in Supplementary Methods V).

In order to describe the more generic setup in Fig. 3a, which now includes two waveguides as well as $N$ nodes, we make use of the SLH input-output formalism as discussed above (see details in Supplementary Methods IV). We write the input and output field operators in the frequency domain as $\hat{b}_{d, j}^{\text {in/out }}(\delta)$, which now contains an additional index $j \in\{$ up, down $\}$ labeling the two waveguides. The single-photon scattering operator for the whole system

$\hat{\mathcal{S}}_{d^{\prime}, d}^{j, i}\left(\nu_{p}, \delta_{p}\right)=\left\langle\operatorname{vac}, \mathcal{G}\left|\hat{b}_{d^{\prime}, j}^{\text {out }}\left(\nu_{p}\right)\left[\hat{b}_{d, i}^{\text {in }}\left(\delta_{p}\right)\right]^{\dagger}\right| \operatorname{vac}, \mathcal{G}\right\rangle$,

where $|\operatorname{vac}, \mathcal{G}\rangle=|\operatorname{vac}\rangle \bigotimes_{n=1}^{N}|G\rangle_{n}$, then contains two additional indices representing the input line $i$ and the output line $j$ of the scattered photon. The derivation and general expression of this operator are provided in the Supplementary Methods IV.

In the ideal case where each GUE scatters photons unidirectionally, the scattering operator factorizes as $\hat{\mathcal{S}}_{L, R}^{, l}\left(\nu_{p}, \delta_{p}\right)=0$ and we obtain

$\hat{\mathcal{S}}_{R, R}^{j, i}\left(\nu_{p}, \delta_{p}\right)=\delta\left(\nu_{p}-\delta_{p}\right) e^{i \tilde{\phi} N}\left[\mathcal{U}_{N} \prod_{n=1}^{N}\left(\hat{S}_{n}\left(\delta_{p}\right) \mathcal{U}_{n-1}\right)\right]_{j, i}$,

with the convention $\prod_{n=1}^{N} A_{n}=A_{N} \ldots A_{1}$, where the propagation phase $\tilde{\phi}=\omega_{0} l / v_{g}$ (with $I$ the distance along the waveguide between two neighbouring nodes [see Fig. 3a]) enters only as a trivial global phase. Here $\mathcal{U}_{n}$ denote the linear optical elements acting on the photonic channels, as shown in Fig. 3a. They can be represented as 2-dimensional unitary matrices acting on a vectorial space which we denote as $\left.\left\{|u p\rangle_{\mathrm{ph}}, \mid \text { down }\right\rangle_{\mathrm{ph}}\right\}$, where the basis vectors $\mid \mathrm{up} /$ down $\rangle_{\mathrm{ph}}$, correspond to the transmission line (either "up" or "down") in which the photon propagates. On this vectorial space the objects $\hat{S}_{n}(\delta)$ are diagonal matrices of qubit operators, which represent the photon scattering on each node. They are defined as $\hat{S}_{n}\left(\delta_{p}\right) \mid$ down $\rangle_{\mathrm{ph}}=\mid$ down $\rangle_{\mathrm{ph}}$ and $\hat{S}_{n}\left(\delta_{p}\right)|\mathrm{up}\rangle_{\mathrm{ph}}=|\mathrm{up}\rangle_{\mathrm{ph}} \hat{\sigma}^{n}\left(\delta_{p}\right)$ as expressed in Eq. (16).
The operator $\hat{S}_{n}\left(\delta_{p}\right)$ thus realizes a frequency-dependent controlled-phase gate between the propagating photon as a "flying" control qubit with states $\mid$ down $\rangle_{\mathrm{ph}}$ and $|\mathrm{up}\rangle_{\mathrm{ph}}$, and qubit atom $n$. For the applications discussed in the following the parameter $\Delta^{n}$ will always be chosen such that the effective interaction in $\hat{S}_{n}\left(\delta_{p}=0\right)$, between a resonant photon and qubit atom $n$, is either trivial (with $\Delta^{n} \gg \gamma_{r}$ ), or realizes a controlled-Z gate $\mid$ down $\rangle_{\mathrm{ph}}\langle\text { down }|+| \text { up }\rangle_{\mathrm{ph}}\langle\operatorname{up}| \hat{\sigma}_{z}^{n} \quad\left(\right.$ with $\left.\Delta^{n}=-\gamma_{r} / 2\right)$ as represented in Fig. $3 d$.

The entanglement structure of the scattering operator $\hat{\mathcal{S}}_{R, R}^{j, i}\left(\nu_{p}, \delta_{p}\right)$ in Eq. (18) is that of a matrix product operator ${ }^{80}$ with bond dimension 2, which is a consequence of quantum information being carried in the network by a propagating photonic qubit. The photon scattering will thus generate entanglement in the qubit array, which can be used e.g. to prepare it in a matrix product state ${ }^{80}$ such as a GHZ state or 1D cluster state $^{81}$ (see details in Supplementary Methods VI). We note that this bond dimension, i.e., the amount of entanglement generatable by scattering a photon in the system, can in principle be increased by expanding the dimensionality of the photonic Hilbert space, e.g. by adding more waveguides.

As a first illustration of the working principles of this passive architecture, we consider one of the most basic protocols requiring quantum information routing, namely quantum state transfer between two stationary qubits. Here, the goal is to transfer a superposition state from one qubit atom, e.g. with $n=1$, to another (possibly distant) one, e.g. with $n=N$, as represented in Fig. 4a. This is achieved by engineering the effective photon-qubit interaction in such a way that the scattering operator in Eq. (18) realizes an effective controlled- $Z$ gate between the distant qubits, thereby enabling universal quantum computation in our architecture. The corresponding protocol circuit is represented in Fig. $4 \mathrm{~b}$, which shows how the initial state of qubit $1|\psi\rangle_{q, 1}=c_{0}|0\rangle_{q, 1}+$ $c_{1}|1\rangle_{q, 1} \quad$ (with $\left|c_{0}\right|^{2}+\left|c_{1}\right|^{2}=1$ ) is transferred as $|\psi\rangle_{q, N}$ upon detection of the photon at the output, while quantum information is erased from qubit 1 . Here $\hat{\sigma}_{z}$ gates are applied conditional on the measurement of the photonic qubit in state $|u p\rangle_{\mathrm{ph}}$, and of qubit 1 in state $|1\rangle_{q, 1}$. The Hadamard gates are defined for the atomic qubits as $\hat{\mathcal{H}}=|+\rangle_{q, n}\langle 0|+|-\rangle_{q, n}\langle 1|$, and are similarly defined for the photonic qubit by replacing $|0 / 1\rangle_{q, n}$ with $\mid$ down/up $\rangle_{\text {ph }}$.

Assuming perfect control over the other parameters of the system, the average fidelity for the quantum state transfer protocol, as defined in Methods, will depend on the photon frequency distribution $f\left(\delta_{p}\right)$ as $\overline{\mathcal{F}}_{\mathrm{QST}}=\int d \delta_{p}\left|f\left(\delta_{p}\right)\right|^{2} \mathcal{F}_{\mathrm{QST}}\left(\delta_{p}\right)$, where $\mathcal{F}_{\mathrm{QST}}\left(\delta_{p}\right)=1-2\left(\delta_{p} / \gamma_{r}\right)^{2}+\mathcal{O}\left(\delta_{p} / \gamma_{r}\right)^{3}$. This sets a bound to the bandwidth $\Delta \omega$ of $f\left(\delta_{p}\right)$ as $\Delta \omega \ll \gamma_{r}$, and thus to the duration $T$ of the protocol as $T \geq 1 / \Delta \omega$ (see below). Standard strategies for heralded quantum communication ${ }^{82}$ can be translated to our protocol in Fig. 4a, by adding ancillary stationary qubits to each node as quantum state "backups", thus enabling quantum communication with high fidelity, even with photon losses due for instance to amplitude attenuation in the waveguides or imperfect photon detection (see Methods). We note that, as discussed above, the photon detection can also be realized using additional nodes as detectors.

As a second application of our architecture for quantum networking, we now show that the setup of Fig. 3a allows to perform entangling operations on many stationary qubits, and can be used to passively probe and measure many-body operators, such as stabilizers of stabilizer codes for quantum error correction $^{35}$. A standard approach for measuring such stabilizer operators consists in entangling the qubits with an ancilla using two-body interactions; the stabilizers can then be accessed by measuring the ancilla ${ }^{83-85}$. Building on a previous protocol for measuring the parity of a pair of quantum dots as unidirectional 
emitters $^{86}$, the measurement of stabilizers is achieved here using an interferometric setup with photonic qubits as ancillas, where the only non-trivial operations on the photons are $\mathcal{U}_{0}=\mathcal{U}_{N}=\hat{\mathcal{H}}$, and one obtains for the scattering operator of Eq. (18)

$\hat{\mathcal{S}}_{R, R}^{j, \text { down }}\left(\nu_{p}, \delta_{p}\right)=\delta\left(\nu_{p}-\delta_{p}\right) e^{i \tilde{\phi} N} \frac{\mathbb{1}+(-1)^{\delta_{j, \text { down }}} \prod_{n} \hat{\sigma}^{n}\left(\delta_{p}\right)}{2}$.

We recall that, with the parameters discussed above, for each stationary qubit $n$ we chose the parameters of the system such that the operator $\hat{\sigma}^{n}\left(\delta_{p}\right)$ is either the identity operator $\mathbb{1}$ or the Pauli operator $\hat{\sigma}_{z}^{n}$ when $\delta_{p}=0$. Defining an arbitrary subset $\mathcal{I}$ of the qubit array, the operator in Eq. (19) can thus be applied to entangle the state of the output photonic qubit (given by the index $j$ ) with the parity $\hat{\mathcal{P}}_{\mathcal{I}}=\prod_{n \in \mathcal{I}} \hat{\sigma}_{z}^{n}$ of the interacting qubits, which can then be measured by detecting the photon. More generally, allowing local unitary operations to be performed on the stationary qubits before and after the scattering enables the measurement of any operator of the form $\prod_{n \in \mathcal{I}} \hat{\boldsymbol{\sigma}}^{n}$, where $\hat{\boldsymbol{\sigma}}^{n}$ is an arbitrary rotation of $\hat{\sigma}_{z}^{n}$ on the Bloch sphere. Examples of such operators are the stabilizers of cluster states, which are universal resources for quantum computation ${ }^{87}$, and of stabilizer codes, where logical qubits are redundantly encoded in many physical qubits and protected by topology 35 .

Despite tremendous recent experimental progress towards measuring stabilizer operators in superconducting platforms ${ }^{88-94}$, realizing stabilizer codes with code distances (i.e., the number of physical qubits) beyond a few qubits remains a great challenge. As we show in the following, our architecture offers a naturally scalable approach to passively probe stabilizers, and thus generate and manipulate stabilizer codes. As an example of stabilizer code, we consider the toric code ${ }^{95}$, where qubits are located on edges of a lattice with periodic boundary conditions. A minimal instance with $N=8$ qubits is represented in Fig. 5 a. The toric code has two types of stabilizers: for each plaquette $p$ and each vertex $v$ of the lattice we associate the stabilizers $\hat{A}_{p}=\prod_{n \in p} \hat{\sigma}_{z}^{n}$ and $\hat{B}_{v}=\prod_{n \in v} \hat{\sigma}_{x}^{n}$, with $\hat{\sigma}_{x}^{n}=|0\rangle_{q, n}\langle 1|+| 1\rangle_{q, n}\langle 0|$. The logical subspace for encoding quantum information then consists of the four states which are eigenstates of all these stabilizers, with eigenvalue +1 . A protocol for preparing the system in one of these four states consists in initializing all qubits in state $\bigotimes_{n}|+\rangle_{q, n}$. The plaquette operators $\hat{A}_{p}$ are then sequentially measured, and the system can be brought to

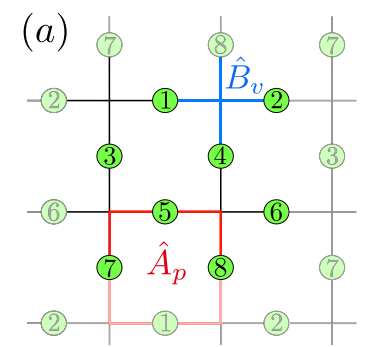

(b)
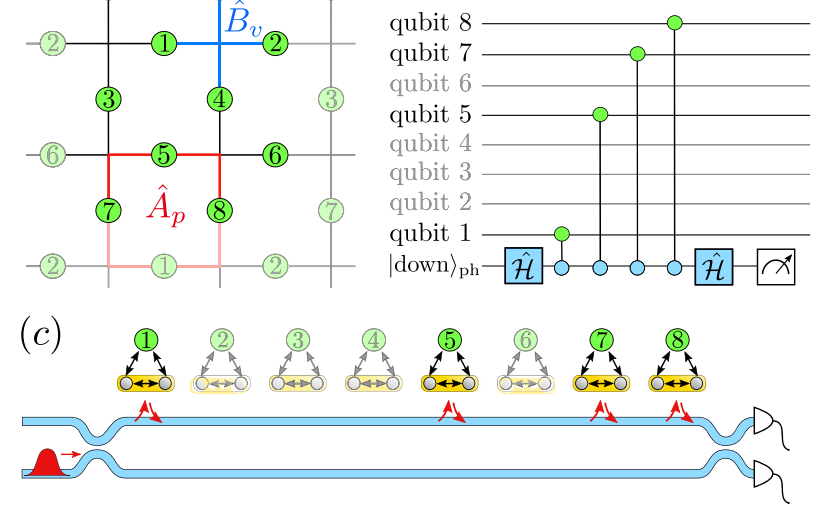

Fig. 5 Toric code generation and manipulation. a Abstract representation of a toric code, where qubits are located on the edges of a 2D lattice with periodic boundary conditions, with here $N=8$ qubits. The two types of stabilizers $\hat{A}_{p}$ and $\hat{B}_{v}$ are represented. b Quantum circuit realizing a measurement of the stabilizer $\hat{A}_{p}$ represented in (a), and c corresponding interferometric setup, with the detunings $\Delta^{n}$ of the GUEs chosen such that only nodes $1,5,7$ and 8 are resonant with the photon. the desired state by applying single-qubit $\hat{\sigma}_{x}^{n}$ gates afterwards, conditioned on the measurement outcomes (see Methods).

In Fig. 5b, c we represent the quantum circuit and the setup realizing the measurement of the operator $\hat{A}_{p}$ shown in Fig. $5 a$. Similar protocols, realized by scattering single photons, can be devised for (i) transferring a superposition state from a single additional stationary qubit to a logical quantum superposition state of the stabilizer code, as well as the reverse process, and (ii) realizing arbitrary logical qubit gates on the code subspace, as well as exponentiated string operators for quantum simulation of anyonic ${ }^{84}$ and fermionic models ${ }^{96}$ (see details in Supplementary Methods VII).

In order to quantify the efficiency of our scheme, we consider the task of performing a measurement of the parity operator $\hat{\mathcal{P}}_{\mathcal{I}}$ on $n_{G}=|\mathcal{I}|$ qubits, with the qubits initially prepared in state $\left|\Psi_{+}\right\rangle=\bigotimes_{n}|+\rangle_{q, n}$. Ideally, detecting the photon at the output of waveguide "up" or "down" projects this state to state $\left|\psi_{\text {up }}^{\text {ideal }}\right\rangle=$ $\frac{1}{\sqrt{2}}\left(\mathbb{1}+\hat{\mathcal{P}}_{\mathcal{I}}\right)\left|\Psi_{+}\right\rangle$or $\left|\Psi_{\text {down }}^{\text {ideal }}\right\rangle=\frac{1}{\sqrt{2}}\left(\mathbb{1}-\hat{\mathcal{P}}_{\mathcal{I}}\right)\left|\Psi_{+}\right\rangle$, respectively. The average fidelity of this process, defined in Methods, takes here the expression $\overline{\mathcal{F}}_{\mathcal{Z}}=\int d \delta_{p}\left|f\left(\delta_{p}\right)\right|^{2} \mathcal{F}_{\mathcal{Z}}\left(\delta_{p}\right)$, with

$\mathcal{F}_{\mathcal{Z}}\left(\delta_{p}\right)=\sum_{j}\left|\left\langle\psi_{j}^{\text {ideal }}\left|\int d \nu_{p} \hat{\mathcal{S}}_{R, R}^{j \text { down }}\left(\nu_{p}, \delta_{p}\right)\right| \Psi_{+}\right\rangle\right|^{2}$,

which we represent in Fig. 6. In Fig. 6a, b we show this fidelity in situations where the photon scattering is not perfectly unidirectional, with the explicit expression of the scattering operator $\hat{\mathcal{S}}_{d^{\prime}, R}^{j \text {, down }}\left(\nu_{p}, \delta_{p}\right)$ from Eq. (17) provided in the Supplementary Methods IV. In these cases where the dynamics is not purely cascaded, the fidelity also depends on the propagation

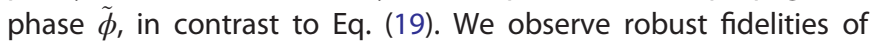
$\mathcal{F}_{\mathcal{Z}}\left(\delta_{p}\right) \gtrsim 99 \%$ for small fluctuations of $V_{1,2}^{n}$ below $\sim 2 \%$ and $J$ below $\sim 5 \%$ around their optimal values. Figure $6 c$, $d$ represents situations where the photon scatters unidirectionally on each node, and shows that the infidelity $1-\mathcal{F}_{\mathcal{Z}}\left(\delta_{p}\right)$ scales quadratically with the deviation of $V$ around $\gamma_{r}$, with the number of interacting qubits $n_{G}$, and with the detuning of the photon $\delta_{p}$.

As an estimation of experimentally achievable performances, we consider $V=\gamma_{r}=2 \pi \times 50 \mathrm{MHz}$. From Fig. $6 \mathrm{~d}$, the gate infidelity intrinsic to our protocol remains below $1 \%$ as long as the photon detuning is below $\left|\delta_{p}\right| \lesssim 0.1 \gamma_{r} / n_{G}$. This sets a bound to the duration $T$ of a stabilizer measurement, as the bandwidth $\Delta \omega$ of the photon frequency distribution $f\left(\delta_{p}\right)$ must satisfy $T \Delta \omega \geq 1$. For instance, assuming the photon wavepacket has a truncated gaussian temporal distribution, we obtain an average fidelity $\overline{\mathcal{F}_{\mathcal{Z}}}$ above $99 \%$ with $T=400$ ns for $n_{G}=4$ (see Methods). All 6 independent stabilizers of the toric code with $N=8$ qubits can then be measured sequentially in a total time $\gtrsim 2.4 \mu \mathrm{s}$. We note that measurements of several stabilizers involving non-overlapping subsets of qubits can be performed in parallel using frequencymultiplexing techniques, as the frequency of their respective GUEs can be tuned to be resonant with probe fields with different frequencies. Measuring simultaneously multiple independent stabilizer operators, by using a different probing frequency for each stabilizer, allows to scale up stabilizer codes without increasing the total measurement time.

\section{DISCUSSION}

To conclude, we presented the design of a unidirectional artificial atom, and demonstrated its application as an on-chip interface between itinerant photons and stationary qubits. This design can be integrated in a modular architecture of photonic quantum networks, where controllable multi-qubit operations are realized by passively scattering itinerant photons, which we illustrated with 

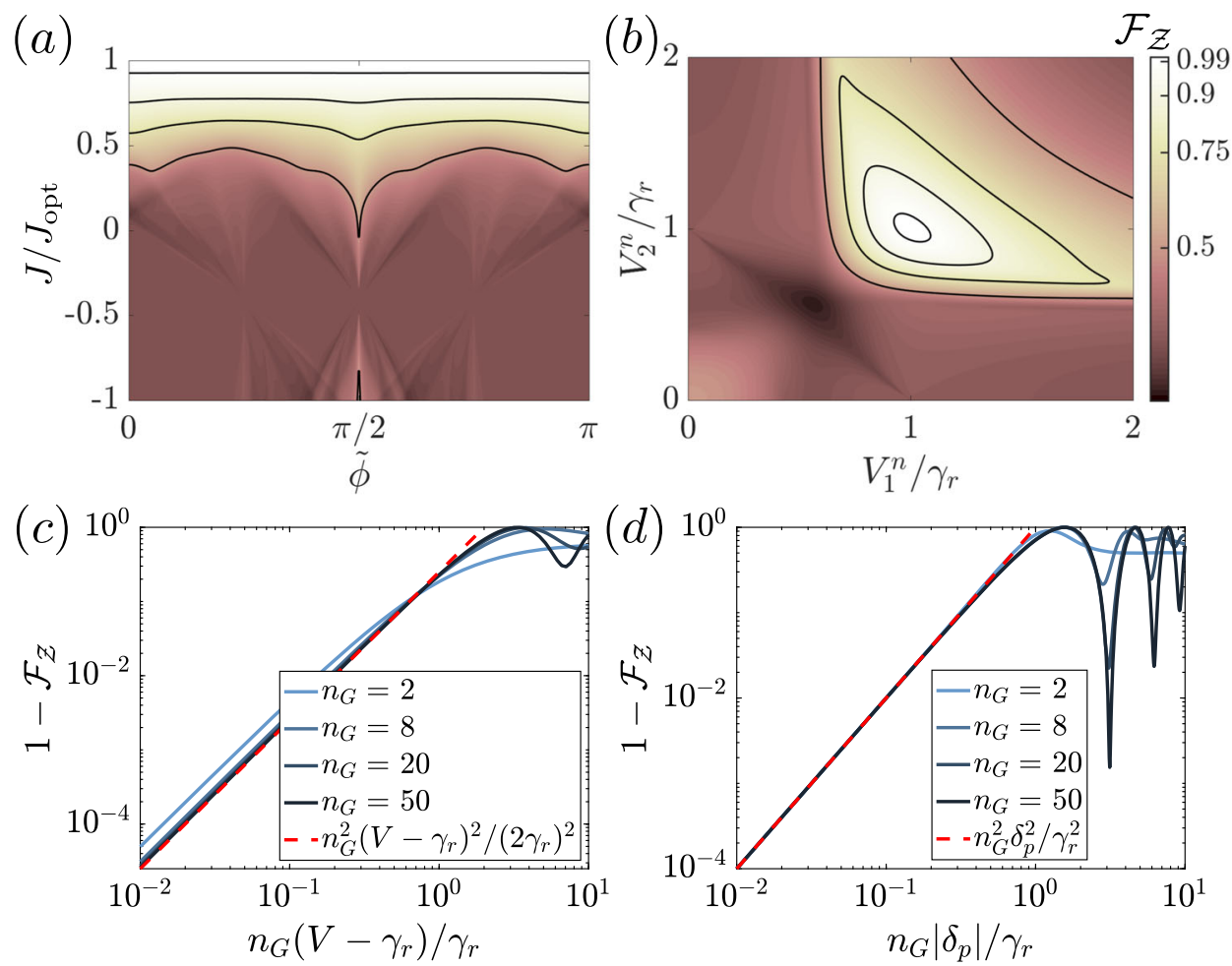

Fig. 6 Stabilizer measurements. Fidelity $\mathcal{F}_{\mathcal{Z}}\left(\delta_{p}\right)$ for the measurement of the parity operator $\hat{\mathcal{P}}_{\mathcal{I}}$ on $n_{G}$ qubits prepared in state $\left|\Psi_{+}\right\rangle$, with $\phi=$ $\phi_{\mathrm{opt}}, \gamma_{1}=\gamma_{2}, r_{k}=0$ and $\Delta^{n}=-\gamma_{r} / 2 . \mathbf{a} V_{1}^{n}=V_{2}^{n}=\gamma_{r}, \delta_{p}=0, n_{G}=4 . \mathbf{b}-\mathbf{d} J=J_{\mathrm{opt}}, \tilde{\phi}=0 . \mathbf{b} \delta_{p}=0, n_{G}=4 . \mathbf{c} \delta_{p}=0, V_{1}^{n}=V_{2}^{n}=V . \mathbf{d} V_{1}^{n}=V_{2}^{n}=\gamma_{r}$.

the realization of quantum state transfer protocols with high fidelity, as well as the measurement of many-body stabilizer operators, pertinent for topological quantum error correction.

In contrast to standard strategies for routing quantum information between nodes of a quantum network, our approach does not make use of circulators. In fact, rather than breaking Lorentz reciprocity for the electromagnetic field (i.e., the invariance under the exchange of source and detector) to control and route an itinerant quantum signal, here the propagation of the quantum signal is set by the itinerant photons injected in the network. This allows to achieve an effective non-reciprocal interaction between stationary qubits with a rather simple design, and an architecture resilient to noise and perturbations.

\section{METHODS}

Superconducting circuit implementation of unidirectional emitters The circuit implementing the GUE is represented in Fig. 1c, and consists of two transmons interacting via a SQUID and coupled at two points to an open transmission line. Following standard quantization procedures $s^{44,45,97}$, we decompose the transmission line, with inductance and capacitance per unit length $I_{0}$ and $c_{0}$, into segments of finite lengths $\Delta x$, and write the Lagrangian of the system as $L=\frac{1}{2} \dot{\boldsymbol{\varphi}}^{\top} \overline{\bar{C}} \dot{\boldsymbol{\varphi}}-V$, where $\boldsymbol{\varphi}=$ $\left(\varphi_{1}, \varphi_{2}, \varphi_{\mathrm{TL}, 1}, \varphi_{\mathrm{TL}, 2}, \varphi_{\mathrm{TL}, 3}, \ldots\right)^{T}$ contains the generalized flux variables associated to the transmons $\left(\varphi_{1}\right.$ and $\left.\varphi_{2}\right)$, and to each segment of the transmission line $\left(\varphi_{\mathrm{TL}, i}\right)$, indexed from left to right. Denoting the indices for the segments coupled to each transmon as $i_{1}$ and $i_{2}$, the capacitance matrix reads $\overline{\bar{C}}=\left(\begin{array}{cc}\overline{\bar{C}}_{a} & -\overline{\bar{C}}_{a, \mathrm{TL}} \\ -\overline{\bar{C}}_{a, \mathrm{TL}}^{T} & \overline{\bar{C}}_{\mathrm{TL}}\end{array}\right)$, with

$\overline{\bar{C}}_{a}=\left(\begin{array}{cc}C_{1}+\bar{C}+c_{1}^{\prime} & -\bar{C} \\ -\bar{C} & C_{2}+\bar{C}+c_{2}^{\prime}\end{array}\right)$,

$\left(\overline{\bar{C}}_{\mathrm{TL}}\right)_{j, k}=\delta_{j, k}\left(c_{0} \Delta x+\delta_{j, 1_{1}} c_{1}^{\prime}+\delta_{j, i_{2}} c_{2}^{\prime}\right)$, and $\left(\overline{\bar{C}}_{a, \mathrm{TL}}\right)_{j, k}=c_{1}^{\prime} \delta_{j, 1} \delta_{k, i_{1}}+c_{2}^{\prime} \delta_{j, 2} \delta_{k, i_{2}}$.
The potential energy, on the other hand, reads

$$
\begin{aligned}
V= & \frac{1}{21_{0} \Delta x} \sum_{i}\left(\varphi_{\mathrm{TL}, \mathrm{i}+1}-\varphi_{\mathrm{TL}, \mathrm{i}}\right)^{2}-\bar{E}_{J} \cos \left[\left(\varphi_{2}-\varphi_{1}\right) / \varphi_{0}\right] \\
& -E_{J}^{1} \cos \left(\varphi_{1} / \varphi_{0}\right)-E_{J}^{2} \cos \left(\varphi_{2} / \varphi_{0}\right),
\end{aligned}
$$

with $\varphi_{0}=\hbar / 2 e$ ( $e$ is the elementary charge)

Defining the conjugate variables $\mathbf{Q}=\frac{\partial L}{\partial \dot{\varphi}}=\overline{\bar{C}} \dot{\boldsymbol{\varphi}}$, we obtain the Hamiltonian of the full system

$$
H_{\text {full }}=\mathbf{Q}^{T} \dot{\boldsymbol{\varphi}}-L=\frac{1}{2} \mathbf{Q}^{T} \cdot(\overline{\bar{C}})^{-1} \cdot \mathbf{Q}+V
$$

which can be decomposed into $H_{\text {full }}=H_{a}+H_{\mathrm{ph}}+H_{\text {int }}$, with an atomic term $H_{a}$ a term for the transmission line $H_{\mathrm{ph}}$ and an interaction term $H_{\text {int }}$. For the artificial atoms we obtain

$$
\begin{aligned}
H_{a}= & \frac{1}{2} \sum_{k} Q_{k}^{2}(\overline{\bar{C}})_{k, k}^{-1}-E_{j}^{1} \cos \left(\varphi_{1} / \varphi_{0}\right)-E_{J}^{2} \cos \left(\varphi_{2} / \varphi_{0}\right) \\
& +Q_{1} Q_{2}(\overline{\bar{C}})_{1,2}^{-1}-\bar{E}_{J} \cos \left[\left(\varphi_{2}-\varphi_{1}\right) / \varphi_{0}\right] .
\end{aligned}
$$

We then promote the flux and charge variables to operators satisfying $\left[\hat{\varphi}_{k}, \hat{Q}_{l}\right]=i \delta_{k, l}$, and express the Hamiltonian in terms of bosonic annihilation operators $\hat{a}_{1}$ and $\hat{a}_{2}$, with

$$
\begin{gathered}
\hat{\varphi}_{k}=\varphi_{0}\left(\frac{2 E_{c}^{k}}{E_{J}^{k}}\right)^{1 / 4}\left(\hat{a}_{k}^{\dagger}+\hat{a}_{k}\right), \\
\hat{Q}_{k}=2 e i\left(\frac{E_{J}^{k}}{32 E_{c}^{k}}\right)^{1 / 4}\left(\hat{a}_{k}^{\dagger}-\hat{a}_{k}\right) .
\end{gathered}
$$

Here $E_{C}^{k}=e^{2} / 2 C_{k}^{\text {eff }}$, with $C_{k}^{\text {eff }}=1 /(\overline{\bar{C}})^{-1} \approx C_{k}+C_{k}^{\prime}+\bar{C}$ for $\left(c_{k}^{\prime}, \bar{C}\right) \ll C_{k}$.

The atomic Hamiltonian $\hat{H}_{a}$ then takes the expression of Eq. (1) by expanding the cosine functions in Eq. (24) up to fourth order, in the limit $\left\langle\left|\hat{\varphi}_{k}\right|\right\rangle \ll \varphi_{0}$, which is achieved in the transmon regime $E_{C}^{k} \ll E_{J}^{k}$, and discarding counter-rotating terms in a rotating wave approximation valid for $\bar{C} \ll C_{k}$ and $\bar{E}_{J} \ll E_{\jmath}^{k}$. To estimate the value of the parameters in Eq. (1), we keep only the leading order terms, and find for the transition frequencies $\omega_{k} \approx \sqrt{8 E_{J}^{k} E_{C}^{k}}$, while the anharmonicities read $U_{k} \approx E_{C}^{k}$. The linear interaction terms have a capacitive component $J_{C}$ and an inductive component $J_{l}$ as expressed in Eq. (7). We note that the conditions of $\bar{C} \ll C_{k}$ and $\bar{E}_{J} \ll E_{J}^{k}$ are required here in order to be able to neglect 
counter-rotating terms such as $\left(J_{C}+J_{1}\right)\left(\hat{a}_{1}^{\dagger} \hat{a}_{2}^{\dagger}+\hat{a}_{1} \hat{a}_{2}\right)$. Similar considerations apply for the non-linear cross-Kerr interaction $X$ as expressed in Eq. (8).

For the transmission line Hamiltonian on the other hand, in the limit $\Delta x \rightarrow 0$ the only non-vanishing terms are $H_{\mathrm{ph}}=\int d x\left[\partial_{x} \varphi(x)\right]^{2} / 2 I_{0}+q(x)^{2} / 2 c_{0}$, where $\varphi(x)$ is the flux variable at position $x$ in the waveguide, and $q(x)$ the charge density. We then express these fields in second quantization in terms of the bosonic operators $b_{R}(\omega)$ and $\hat{b}_{L}(\omega)$ as

$$
\begin{gathered}
\hat{\varphi}(x)=\int d \omega \sqrt{\frac{Z_{0}}{4 \pi \omega}}\left(\hat{b}_{R}(\omega) e^{i \omega x / v_{g}}+\hat{b}_{L}(\omega) e^{-i \omega x / v_{g}}\right)+\text { h.c. } \\
\hat{q}(x)=-i \int d \omega \sqrt{\frac{Z_{0} c_{0}^{2} \omega}{4 \pi}}\left(\hat{b}_{R}(\omega) e^{i \omega x / v_{g}}+\hat{b}_{L}(\omega) e^{-i \omega x / v_{g}}\right) \\
+ \text { h.c. }
\end{gathered}
$$

where $v_{g}=1 / \sqrt{I_{0} c_{0}}$ is the group velocity of photons in the transmission line and $Z_{0}=\sqrt{I_{0} / c_{0}}$ the transmission line characteristic impedance, and we obtain $\hat{H}_{\text {ph }}=\int d \omega \omega\left[\hat{b}_{R}^{\dagger}(\omega) \hat{b}_{R}(\omega)+\hat{b}_{L}^{\dagger}(\omega) \hat{b}_{L}(\omega)\right]$.

Finally, for the interaction term we obtain

$\hat{H}_{\text {int }}=\frac{1}{c_{0}}\left(\begin{array}{ll}\hat{Q}_{1} & \hat{Q}_{2}\end{array}\right) \overline{\bar{C}}_{a}^{-1}\left(\begin{array}{l}c_{1}^{\prime} \hat{q}\left(x_{1}\right) \\ c_{2}^{\prime} \hat{q}\left(x_{2}\right)\end{array}\right)$,

where $x_{1}$ and $x_{2}$ denote the position of the two coupling points along the waveguide. We then obtain the expression in Eq. (2) by setting $x_{1}=0$ and $x_{2}=\bar{d}$, redefining the phase of the right-propagating modes as $\hat{b}_{R}(\omega) \rightarrow$ $\hat{b}_{R}(\omega) e^{-i \omega \bar{d} / v_{g}}$ and approximating the couplings to be constant over the relevant bandwidth. Assuming for simplicity $E_{j}^{1} / E_{C}^{1} \approx E_{J}^{2} / E_{C}^{2}$, the coupling rates express as $\gamma_{k}=\left(c_{k}^{\prime} / C_{k}^{\text {eff }}\right)^{2} \omega_{0} e^{2} Z_{0} \sqrt{E_{J}^{k} /\left(8 E_{C}^{k}\right)}$ and the cross-coupling coefficients as $r_{k}=\bar{C} / C_{k}^{\text {eff }}$.

\section{Quantum state transfer protocol}

The average fidelity $\overline{\mathcal{F}}_{\text {QST }}$ for the quantum state transfer protocol is evaluated by applying the protocol on an initially maximally entangled state between qubit 1 and a virtual ancilla qubit (denoted $a)^{98}$ as $\left|\psi_{i}\right\rangle=\left(|0\rangle_{q, 1}|0\rangle_{a}+|1\rangle_{q, 1}|1\rangle_{a}\right)|+\rangle_{q, N} / \sqrt{2}$. After performing the protocol as represented in Fig. 4, we obtain the state of the system of qubit $N$ and ancilla as a density matrix $\hat{\rho}_{f}$. Ideally, the state of qubit $N$ and the ancilla should be pure and entangled as $\left|\Psi_{\text {ideal }}\right\rangle=\left(|0\rangle_{q, N}|0\rangle_{a}+|1\rangle_{q, N}|1\rangle_{a}\right) / \sqrt{2}$. The average fidelity is then defined as $\overline{\mathcal{F}}_{\text {QST }}=\operatorname{Tr}\left[\left\langle\psi_{\text {ideal }}\left|\rho_{\mathrm{f}}\right| \Psi_{\text {ideal }}\right\rangle\right]$.

Here, the state of the system before the scattering of the photon, injected in the system from line "down", reads $\mid$ in $\rangle=\int d \delta_{p} f\left(\delta_{p}\right)\left[\hat{b}_{R \text {,down }}^{\text {in }}\left(\delta_{p}\right)\right]^{\dagger} \mid$ vac, $\left.\mathcal{G}\right\rangle|\Psi\rangle_{i}$, with frequency distribution $f$ $\left(\delta_{p}\right)$. Assuming unidirectional photon - GUE interactions, from the expression in Eq. (18) the state after the scattering reads $\mid$ out $\rangle=\sum_{j} \int d \delta_{p} f\left(\delta_{p}\right)\left[\hat{b}_{R, j}^{\text {out }}\left(\delta_{p}\right)\right]^{\dagger} \mid$ vac, $\left.\mathcal{G}\right\rangle\left|\Psi_{j}\left(\delta_{p}\right)\right\rangle$, where $\left|\Psi_{j}\left(\delta_{p}\right)\right\rangle=$ $\left[\mathcal{H} \hat{S}_{N}\left(\delta_{p}\right) \mathcal{H} \hat{S}_{1}\left(\delta_{p}\right) \mathcal{H}\right]_{j, \text { down }}|\Psi\rangle_{i}$ represents the state of the qubits when the photon is scattered to line $j$. The (unnormalized) qubit density matrix $\hat{\rho}_{j}$, conditioned on the detection of the photon at the output of line $j$, is then obtained as $\hat{\rho}_{j}=\int d \delta_{p}\left|f\left(\delta_{p}\right)\right|^{2}\left|\Psi_{j}\left(\delta_{p}\right)\right\rangle\left\langle\psi_{j}\left(\delta_{p}\right)\right|$.

Denoting all the other operations performed in the circuit of Fig. 4 after the photon scattering and subsequent detection at the output of line $j$, including the projective measurements of qubit atom 1 , as superoperators $\hat{P}_{j}$, we then obtain the reduced density matrix as $\hat{\rho}_{f}=\operatorname{Tr}_{q, 1}\left(\hat{P}_{\text {up }}\left[\hat{\rho}_{\text {up }}\right]+\hat{P}_{\text {down }}\left[\hat{\rho}_{\text {down }}\right]\right)$, where $\operatorname{Tr}_{q, 1}$ denotes the trace over qubit 1. The average fidelity finally expresses as $\overline{\mathcal{F}}_{\mathrm{QST}}=\int d \delta_{p}\left|f\left(\delta_{p}\right)\right|^{2} \mathcal{F}_{\mathrm{QST}}\left(\delta_{p}\right)$, with

$$
\begin{aligned}
\mathcal{F}_{\mathrm{QST}}\left(\delta_{p}\right) & =\frac{\gamma_{r}^{8}-2 \gamma_{r}^{6} \delta_{p}^{2}-2 \gamma_{r}^{5} \delta_{p}^{3}+3 \gamma_{r}^{4} \delta_{p}^{4}+2 \gamma_{r}^{3} \delta_{p}^{5}+4 \delta_{p}^{8}}{\left(\gamma_{r}^{4}+4 \delta_{p}^{4}\right)^{2}} \\
& =1-2\left(\delta_{p} / \gamma_{r}\right)^{2}+\mathcal{O}\left(\delta_{p} / \gamma_{r}\right)^{3} .
\end{aligned}
$$

Including in the description the finite probability $P_{d}$ of losing the photon in the process, due for instance to amplitude attenuation in the waveguides or to a faulty photon detection, the overall transfer fidelity is $\left(1-P_{d}\right) \overline{\mathcal{F}}_{\text {QST. }}$. Standard strategies for quantum error correction can however be applied to correct for such photon losses. For example, following ref. ${ }^{82}$, we can add an ancillary backup stationary qubit $b$ to node 1 and, before performing the state transfer protocol, entangle it with qubit

$$
\begin{aligned}
& 1 \text { as } \\
& \left(\begin{array}{c}
|0\rangle_{q, 1} \\
|1\rangle_{q, 1}
\end{array}\right)|0\rangle_{b}|+\rangle_{q, N} \rightarrow\left(\begin{array}{c}
|0\rangle_{q, 1}|1\rangle_{b}+|1\rangle_{q, 1}|0\rangle_{b} \\
|1\rangle_{q, 1}|1\rangle_{b}+|0\rangle_{q, 1}|0\rangle_{b}
\end{array}\right)|+\rangle_{q, N} .
\end{aligned}
$$

In case the photon is not detected after the scattering, the initial superposition can then be retrieved by measuring qubit 1 , as the photon scattering operator in Eq. (16) is diagonal in the computational basis of the qubits. From Eq. (29), for the measurement outcome $|0\rangle_{q .1}$, the state of the backup qubit is projected to $\left(\begin{array}{c}|1\rangle_{b} \\ |0\rangle_{b}\end{array}\right)$, while the outcome $|1\rangle_{q, 1}$ yields $\left(\begin{array}{l}|0\rangle_{b} \\ |1\rangle_{b}\end{array}\right)$. This allows to prepare the system back to the entangled state (29), and repeat the procedure until the photon is successfully detected at the output, which requires on average $1 /\left(1-P_{d}\right)$ trials. At this stage, the state transfer protocol can resume normally, which transfers the entanglement with the backup qubit $b$ from qubit 1 to qubit $N$, yielding

$$
\left(\begin{array}{c}
|1\rangle_{b}|0\rangle_{q, N}+|0\rangle_{b}|1\rangle_{q, N} \\
|1\rangle_{b}|1\rangle_{q, N}+|0\rangle_{b}|0\rangle_{q, N}
\end{array}\right) \text {. }
$$

The qubit superposition is then finally transferred to qubit $N$ by measuring the backup qubit $b$ and, depending on the outcome, performing a local $\hat{\sigma}_{x}^{N}$ gate on qubit $N$.

\section{Protocol for toric code generation and manipulation}

The toric code is a stabilizer code where physical qubits are located on the edges of a 2D lattice with periodic boundary conditions ${ }^{95}$. The code has two types of stabilizers: as represented in Fig. 5 a, for each plaquette $p$ of the lattice we define an operator $\hat{A}_{p}=\prod_{n \in p} \hat{\sigma}_{z}^{n}$, and, similarly, for each vertex $v$ we define $\hat{B}_{v}=\prod_{n \in v} \hat{\sigma}_{x}^{n}$. With an $N_{l} \times N_{l}$ lattice [e g. $N_{l}=2$ in Fig. 5 a], the number of physical qubits is $N=2 N_{l}^{2}$, while the number of independent stabilizers is $2\left(N_{l}^{2}-1\right)$. Thus, the manifold of states $|\Phi\rangle$ satisfying the constraints $\hat{A}_{p}|\Phi\rangle=|\Phi\rangle$ and $\hat{B}_{v}|\Phi\rangle=|\Phi\rangle$ for all $p$ and $v$ is four-dimensional. One such state can be expressed as

$$
\left|\Phi_{1}\right\rangle=\frac{1}{\sqrt{2}} \prod_{p}\left(\mathbb{1}+\hat{A}_{p}\right) \bigotimes_{n=1}^{N}|+\rangle_{q, n}
$$

which projects $\bigotimes_{n=1}^{N}|+\rangle_{q, n}$ on the eigenmanifold of each $\hat{A}_{p}$ with eigenvalue +1 . In the protocol of Fig. 5 , we prepare state $\left|\Phi_{1}\right\rangle$ by performing sequential measurements of the $\hat{A}_{p}$ operators. From the list of the measurement outcomes, one can always perform single-qubit $\hat{\sigma}_{x}^{n}$ gates such that the state becomes an eigenstate of all $\hat{A}_{p}$ with eigenvalue +1 . Since $\hat{B}_{v} \bigotimes_{n=1}^{N}|+\rangle_{q, n}=\bigotimes_{n=1}^{N}|+\rangle_{q, n}$ for all $v$, and as all the stabilizers commute, the state remains an eigenstate of all $\hat{B}_{v}$ with eigenvalue +1 throughout the protocol, and we prepared the system in state $\left|\Phi_{1}\right\rangle$.

The other three code states can be obtained from $\left|\Phi_{1}\right\rangle$ by applying products of $\hat{\sigma}_{z}^{n}$ gates on all qubits along horizontal or vertical cyclic paths passing through lattice sites. We denote such horizontal and vertical cyclic paths as $\boldsymbol{\gamma}_{1}$ and $\boldsymbol{\gamma}_{2}$ respectively. For the minimal instance represented in Fig. 5a, one can take for instance $\boldsymbol{\gamma}_{1}=\{5,6\}$ and $\boldsymbol{\gamma}_{2}=\{4,8\}$. Conversely, we define vertical and horizontal cyclic paths passing through plaquette centers, which we denote respectively as $\boldsymbol{\gamma}_{1}^{*}$ and $\boldsymbol{\gamma}_{2}^{*}$. In the example of Fig. 5a one can use for instance $\boldsymbol{\gamma}_{1}^{*}=\{1,5\}$ and $\boldsymbol{\gamma}_{2}^{*}=\{3,4\}$. We then define logical operators on the code manifold as the string operators $\hat{Z}_{1}=\prod_{n \in \boldsymbol{Y}_{1}} \hat{\sigma}_{z}^{n}, \hat{X}_{1}=\prod_{n \in \boldsymbol{Y}_{1}} \hat{\sigma}_{x}^{n}, \hat{Z}_{2}=\prod_{n \in \boldsymbol{Y}_{2}} \hat{\sigma}_{z}^{n}$ and $\hat{X}_{2}=\prod_{n \in \boldsymbol{Y}_{2}^{*}} \hat{\sigma}_{x}^{n}$. This allows to define the other three code states as $\left|\Phi_{2}\right\rangle=\hat{Z}_{1}\left|\Phi_{1}\right\rangle,\left|\Phi_{3}\right\rangle=\hat{Z}_{2}\left|\Phi_{1}\right\rangle$ and $\left|\Phi_{4}\right\rangle=\hat{Z}_{2} \hat{Z}_{1}\left|\Phi_{1}\right\rangle$, which satisfy $\hat{X}_{\alpha}\left|\Phi_{\beta}\right\rangle=(-1)^{s_{a, \beta}}\left|\Phi_{\beta}\right\rangle$ with $s_{1,1}=s_{1,3}=s_{2,1}$ $=s_{2,2}=1$ and $s_{1,2}=s_{1,4}=s_{2,3}=s_{2,4}=-1$. We note that in our setup, the logical operators $\hat{Z}_{1,2}$ and $\hat{X}_{1,2}$ are measurable in the very same way as the stabilizers, without having to measure individual physical qubits which would project the state out of the code subspace.

Fidelity of stabilizer measurements with finite photon pulse We consider here the scattering of a photon wavepacket with a temporal distribution given by

$$
f(\tilde{t})=A e^{-t^{2} /\left(4 \sigma_{t}^{2}\right)} \Theta(T / 2-|t|)
$$

where $T$ is the temporal extent of the wavepacket, $\sigma_{t}$ its temporal width, $\Theta$ the Heaviside step function, and $A$ a normalization constant such that 
$\int_{-T / 2}^{T / 2}|f(\tilde{t})|^{2} d t=1$. With the qubits initialized in state $\left|\Psi_{+}\right\rangle$as expressed above, this realizes a scattering with the input state $\mid$ in $\rangle=\int d \delta_{p} f\left(\delta_{p}\right)\left[\hat{b}_{R, \text { down }}^{\text {in }}\left(\delta_{p}\right)\right]^{\dagger}|\operatorname{vac}\rangle\left|\Psi_{+}\right\rangle, \quad$ where $f\left(\delta_{p}\right)=$ $(1 / \sqrt{2 \pi}) \int d t f(\tilde{t}) e^{i \delta_{p} t}$ is the Fourier transform of $f(\tilde{t})$. Without necessarily assuming purely unidirectional photon - GUE interactions, the system is then left after the scattering in state $\mid$ out $\rangle=\sum_{d^{\prime}, j} \int d \delta_{p} f\left(\delta_{p}\right)\left[\hat{b}_{d^{\prime}, j}^{\text {out }}\left(\delta_{p}\right)\right]^{\dagger}|\operatorname{vac}, \mathcal{G}\rangle\left|\Psi_{d^{\prime}, j}\left(\delta_{p}\right)\right\rangle$, where $\left|\Psi_{d^{\prime}, j}\left(\delta_{p}\right)\right\rangle=$ $\int d \nu_{p} \hat{\mathcal{S}}_{d^{\prime}, R}^{j, \text { down }}\left(\nu_{p}, \delta_{p}\right)\left|\Psi_{+}\right\rangle$, and we made use of the fact that the singlephoton scattering operator is proportional to $\delta\left(v_{p}-\delta_{p}\right)$ (with its general expression provided in the Supplementary Methods IV).

Detecting the photon at the right output of waveguide $j$ yields for the qubits the (unnormalized) density matrix $\hat{\rho}_{j}=\int d \delta_{p}\left|f\left(\delta_{p}\right)\right|^{2}\left|\Psi_{R, j}\left(\delta_{p}\right)\right\rangle\left\langle\Psi_{R, j}\left(\delta_{p}\right)\right|$. The measurement fidelity is then defined as $\overline{\mathcal{F}}_{\mathcal{Z}}=\overline{\mathcal{F}}_{\text {down }}+\overline{\mathcal{F}}_{\text {up }}$ with

$\overline{\mathcal{F}_{j}}=\left\langle\psi_{j}^{\text {ideal }}\left|\hat{\rho}_{j}\right| \psi_{j}^{\text {ideal }}\right\rangle=\int d \delta_{p}\left|f\left(\delta_{p}\right)\right|^{2} \mathcal{F}_{\mathcal{Z}}\left(\delta_{p}\right)$,

with $\mathcal{F}_{\mathcal{Z}}\left(\delta_{p}\right)$ as expressed in Eq. (20).

\section{Note added in proof}

We recently became aware of related unpublished work by N. Gheeraert, S. Kono and Y. Nakamura (personal communication).

\section{DATA AVAILABILITY}

The data sets generated during the current study are available from the corresponding author on request.

\section{CODE AVAILABILITY}

All the numerical data presented in this paper are results of Matlab simulations conducted by POG. The code used to generate this data will be made available to the interested reader upon request.

Received: 6 November 2019; Accepted: 24 February 2020; Published online: 27 March 2020

\section{REFERENCES}

1. Devoret, M. H. \& Schoelkopf, R. J. Superconducting circuits for quantum information: an outlook. Science 339, 1169-1174 (2013).

2. Gambetta, J. M., Chow, J. M. \& Steffen, M. Building logical qubits in a superconducting quantum computing system. npj Quantum Inf. 3, 2 (2017).

3. Wendin, G. Quantum information processing with superconducting circuits: a review. Rep. Prog. Phys. 80, 106001 (2017).

4. Krantz, P. et al. A quantum engineeras guide to superconducting qubits. Appl. Phys. Rev. 6, 21318 (2019).

5. Kimble, H. J. The quantum internet. Nature 453, 1023 (2008)

6. Gu, X., Kockum, A. F., Miranowicz, A., Liu, Y.-x \& Nori, F. Microwave photonics with superconducting quantum circuits. Phys. Rep. 718-719, 1-102 (2017).

7. Hogan, C. L. The ferromagnetic Faraday effect at microwave frequencies and its applications. Rev. Mod. Phys. 25, 253-262 (1953).

8. Caloz, C. et al. Electromagnetic nonreciprocity. Phys. Rev. Appl. 10, 47001 (2018)

9. Kamal, A., Clarke, J. \& Devoret, M. H. Noiseless non-reciprocity in a parametric active device. Nat. Phys. 7, 311 (2011).

10. Abdo, B. et al. Josephson directional amplifier for quantum measurement of superconducting circuits. Phys. Rev. Lett. 112, 167701 (2014).

11. Estep, N. A., Sounas, D. L., Soric, J. \& Alù, A. Magnetic-free non-reciprocity and isolation based on parametrically modulated coupled-resonator loops. Nat. Phys. 10, 923 (2014).

12. Kerckhoff, J., Lalumière, K., Chapman, B. J., Blais, A. \& Lehnert, K. W. On-chip superconducting microwave circulator from synthetic rotation. Phys. Rev. Appl. 4 , 34002 (2015).

13. Sliwa, K. M. et al. Reconfigurable Josephson circulator/directional amplifier. Phys. Rev. X 5, 41020 (2015).

14. Roushan, $P$. et al. Chiral ground-state currents of interacting photons in a synthetic magnetic field. Nat. Phys. 13, 146 (2016).

15. Barzanjeh, S. et al. Mechanical on-chip microwave circulator. Nat. Commun. 8, 953 (2017).
16. Chapman, B. J. et al. Widely tunable on-chip microwave circulator for superconducting quantum circuits. Phys. Rev. X 7, 41043 (2017).

17. Kamal, A. \& Metelmann, A. Minimal models for nonreciprocal amplification using biharmonic Drives. Phys. Rev. Appl. 7, 34031 (2017).

18. Metelmann, A. \& Türeci, H. E. Nonreciprocal signal routing in an active quantum network. Phys. Rev. A 97, 43833 (2018).

19. Koch, J., Houck, A. A., Hur, K. L. \& Girvin, S. M. Time-reversal-symmetry breaking in circuit-QED-based photon lattices. Phys. Rev. A 82, 43811 (2010).

20. Müller, C., Guan, S., Vogt, N., Cole, J. H. \& Stace, T. M. Passive on-chip superconducting circulator using a ring of tunnel junctions. Phys. Rev. Lett. 120, 213602 (2018).

21. Hoi, I.-C. et al. Demonstration of a single-photon router in the microwave regime. Phys. Rev. Lett. 107, 73601 (2011).

22. Neumeier, L., Leib, M. \& Hartmann, M. J. Single-photon transistor in circuit quantum electrodynamics. Phys. Rev. Lett. 111, 63601 (2013).

23. Manzoni, M. T., Reiter, F., Taylor, J. M. \& Sørensen, A. S. Single-photon transistor based on superconducting systems. Phys. Rev. B 89, 180502 (2014).

24. Ekström, M. K. et al. Surface acoustic wave unidirectional transducers for quantum applications. Appl. Phys. Lett. 110, 73105 (2017).

25. Müller, C., Combes, J., Hamann, A. R., Fedorov, A. \& Stace, T. M. Nonreciprocal atomic scattering: a saturable, quantum Yagi-Uda antenna. Phys. Rev. A 96, 53817 (2017).

26. Hamann, A. R. et al. Nonreciprocity realized with quantum nonlinearity. Phys. Rev. Lett. 121, 123601 (2018).

27. Lodahl, P. et al. Chiral quantum optics. Nature 541, 473 (2017).

28. Kockum, A. F., Delsing, P. \& Johansson, G. Designing frequency-dependent relaxation rates and Lamb shifts for a giant artificial atom. Phys. Rev. A 90, 13837 (2014).

29. Guo, L., Grimsmo, A., Kockum, A. F., Pletyukhov, M. \& Johansson, G. Giant acoustic atom: a single quantum system with a deterministic time delay. Phys. Rev. A 95, 53821 (2017).

30. Kockum, A. F., Johansson, G. \& Nori, F. Decoherence-Free Interaction between Giant Atoms in Waveguide Quantum Electrodynamics. Phys. Rev. Lett. 120, 140404 (2018).

31. Andersson, G., Suri, B., Guo, L., Aref, T. \& Delsing, P. Non-exponential decay of a giant artificial atom. Nat. Phys. 15, 1123-1127 (2019).

32. Guo, L., Kockum, A. F., Marquardt, F. \& Johansson, G. Oscillating bound states for a giant atom. Preprint at https://arxiv.org/abs/1911.13028 (2019).

33. Kannan, B. et al. Waveguide quantum electrodynamics with giant superconducting artificial atoms. Preprint at https://arxiv.org/abs/1912.12233 (2019).

34. Kockum, A. F. Quantum optics with giant atoms-the first five years. Preprint at https://arxiv.org/abs/1912.13012 (2019).

35. Gottesman, D. Stabilizer Codes and Quantum Error Correction. Ph.D. thesis (California Institute of Technology, 1997).

36. Gardiner, C. \& Zoller, P. Quantum Noise: A Handbook of Markovian and NonMarkovian Quantum Stochastic Methods with Applications to Quantum Optics. Springer Series in Synergetics (Springer, 2004).

37. Koch, J. et al. Charge-insensitive qubit design derived from the Cooper pair box. Phys. Rev. A 76, 42319 (2007).

38. Chen, Y. et al. Qubit architecture with high coherence and fast tunable coupling. Phys. Rev. Lett. 113, 220502 (2014).

39. Kounalakis, M., Dickel, C., Bruno, A., Langford, N. K. \& Steele, G. A. Tuneable hopping and nonlinear cross-Kerr interactions in a high-coherence superconducting circuit. npj Quantum Inf. 4, 38 (2018).

40. Collodo, M. C. et al. Observation of the crossover from photon ordering to delocalization in tunably coupled resonators. Phys. Rev. Lett. 122, 183601 (2019).

41. Jin, J., Rossini, D., Fazio, R., Leib, M. \& Hartmann, M. J. Photon solid phases in driven arrays of nonlinearly coupled cavities. Phys. Rev. Lett. 110, 163605 (2013).

42. Sameti, M., Potočnik, A., Browne, D. E., Wallraff, A. \& Hartmann, M. J. Superconducting quantum simulator for topological order and the toric code. Phys. Rev. A 95, 42330 (2017).

43. Marcos, D. et al. Two-dimensional lattice gauge theories with superconducting quantum circuits. Ann. Phys. 351, 634 (2014).

44. Johansson, G., Tornberg, L., Shumeiko, V. S. \& Wendin, G. Readout methods and devices for Josephson-junction-based solid-state qubits. J. Phys.: Condens. Matter 18, 5901 (2006).

45. Lalumière, K. et al. Input-output theory for waveguide QED with an ensemble of inhomogeneous atoms. Phys. Rev. A 88, 43806 (2013).

46. Gardiner, C. W. Driving a quantum system with the output field from another driven quantum system. Phys. Rev. Lett. 70, 2269 (1993).

47. Carmichael, H. J. Quantum trajectory theory for cascaded open systems. Phys. Rev. Lett. 70, 2273 (1993).

48. Metelmann, A. \& Clerk, A. A. Nonreciprocal photon transmission and amplification via reservoir engineering. Phys. Rev. X 5, 21025 (2015).

49. Junge, C., O'Shea, D., Volz, J. \& Rauschenbeutel, A. Strong coupling between single atoms and nontransversal photons. Phys. Rev. Lett. 110, 213604 (2013).

50. Mitsch, R., Sayrin, C., Albrecht, B., Schneeweiss, P. \& Rauschenbeutel, A. Quantum state-controlled directional spontaneous emission of photons into a nanophotonic waveguide. Nat. Commun. 5, 5713 (2014). 
51. Shomroni, I. et al. All-optical routing of single photons by a one-atom switch controlled by a single photon. Science 345, 903 (2014).

52. Bechler, O. et al. A passive photon-atom qubit swap operation. Nat. Phys. 14, 996 (2018).

53. Söllner, l. et al. Deterministic photon-emitter coupling in chiral photonic circuits. Nat. Nanotechnol. 10, 775 (2015).

54. Coles, R. J. et al. Chirality of nanophotonic waveguide with embedded quantum emitter for unidirectional spin transfer. Nat. Commun. 7, 11183 (2016).

55. Barik, S. et al. A topological quantum optics interface. Science 359, 666 (2018).

56. Barik, S., Karasahin, A., Mittal, S., Waks, E. \& Hafezi, M. Chiral quantum optics using a topological resonator. Preprint at https://arxiv.org/abs/1906.11263 (2019).

57. Bliokh, K. Y., Rodríguez-Fortuño, F. J., Nori, F. \& Zayats, A. V. Spin-orbit interactions of light. Nat. Photonics 9, 796 (2015).

58. Ramos, T., Vermersch, B., Hauke, P., Pichler, H. \& Zoller, P. Non-Markovian dynamics in chiral quantum networks with spins and photons. Phys. Rev. A 93, 62104 (2016).

59. Vermersch, B., Ramos, T., Hauke, P. \& Zoller, P. Implementation of chiral quantum optics with Rydberg and trapped-ion setups. Phys. Rev. A 93, 63830 (2016).

60. Grankin, A., Guimond, P. O., Vasilyev, D., Vermersch, B. \& Zoller, P. Free-space photonic quantum link and chiral quantum optics. Phys. Rev. A 98, 43825 (2018).

61. Hoi, I.-C. et al. Microwave quantum optics with an artificial atom in onedimensional open space. New J. Phys 15, 25011 (2013).

62. Stannigel, K., Rabl, P. \& Zoller, P. Driven-dissipative preparation of entangled states in cascaded quantum-optical networks. New J. Phys 14, 63014 (2012).

63. Ramos, T., Pichler, H., Daley, A. \& Zoller, P. Quantum spin dimers from chiral dissipation in cold-atom chains. Phys. Rev. Lett. 113, 237203 (2014).

64. Pichler, H., Ramos, T., Daley, A. J. \& Zoller, P. Quantum optics of chiral spin networks. Phys. Rev. A 91, 1-19 (2015).

65. Gough, J. \& James, M. R. Quantum feedback networks: hamiltonian formulation. Commun. Math. Phys. 287, 1109 (2009).

66. Gough, J. \& James, M. R. The series product and its application to quantum feedforward and feedback networks. IEEE Trans. Autom. Control 54, 2530 (2009).

67. Combes, J., Kerckhoff, J. \& Sarovar, M. The SLH framework for modeling quantum input-output networks. Adv. Phys.: X 2, 784 (2017).

68. Pichler, H. \& Zoller, P. Photonic circuits with time delays and quantum feedback. Phys. Rev. Lett. 116, 93601 (2016).

69. Gleyzes, S. et al. Quantum jumps of light recording the birth and death of a photon in a cavity. Nature 446, 297 (2007).

70. Johnson, B. R. et al. Quantum non-demolition detection of single microwave photons in a circuit. Nat. Phys. 6, 663 (2010).

71. Narla, A. et al. Robust concurrent remote entanglement between two superconducting qubits. Phys. Rev. X 6, 31036 (2016).

72. Kono, S., Koshino, K., Tabuchi, Y., Noguchi, A. \& Nakamura, Y. Quantum nondemolition detection of an itinerant microwave photon. Nat. Phys. 14, 546 (2018).

73. Besse, J.-C. et al. Single-shot quantum nondemolition detection of individual itinerant microwave photons. Phys. Rev. X 8, 21003 (2018).

74. Fan, B. et al. Breakdown of the Cross-Kerr Scheme for Photon Counting. Phys. Rev. Lett. 110, 53601 (2013).

75. Hoi, I.-C. et al. Giant Cross-Kerr Effect for Propagating Microwaves Induced by an Artificial Atom. Phys. Rev. Lett. 111, 53601 (2013).

76. Sathyamoorthy, S. R. et al. Quantum Nondemolition Detection of a Propagating Microwave Photon. Phys. Rev. Lett. 112, 93601 (2014).

77. Hadfield, R. H. Single-photon detectors for optical quantum information applications. Nat. Photonics 3, 696 (2009)

78. Inomata, K. et al. Single microwave-photon detector using an artificial $\Lambda$-type three-level system. Nat. Commun. 7, 12303 (2016).

79. Sathyamoorthy, S. R., Stace, T. M. \& Johansson, G. Detecting itinerant single microwave photons. Comptes Rendus Phys. 17, 756 (2016).

80. Schollwöck, U. The density-matrix renormalization group in the age of matrix product states. Ann. Phys. 326, 96 (2011).

81. Briegel, H. J. \& Raussendorf, R. Persistent entanglement in arrays of interacting particles. Phys. Rev. Lett. 86, 910 (2001)

82. van Enk, S. J., Cirac, J. I. \& Zoller, P. Ideal quantum communication over noisy channels: a quantum optical implementation. Phys. Rev. Lett. 78, 4293 (1997)

83. Aguado, M., Brennen, G. K., Verstraete, F. \& Cirac, J. I. Creation, manipulation, and detection of abelian and non-abelian anyons in optical lattices. Phys. Rev. Lett. 101, 260501 (2008).

84. Jiang, L. et al. Anyonic interferometry and protected memories in atomic spin lattices. Nat. Phys. 4, 482 (2008).

85. Müller, M., Hammerer, K., Zhou, Y. L., Roos, C. F. \& Zoller, P. Simulating open quantum systems: from many-body interactions to stabilizer pumping. New J. Phys. 13, 85007 (2011).

86. Mahmoodian, S., Lodahl, P. \& Sørensen, A. S. QuanTum Networks With Chirallight-matter Interaction In Waveguides. Phys. Rev. Lett. 117, 240501 (2016).

87. Briegel, H. J., Browne, D. E., Dür, W., Raussendorf, R. \& Van den Nest, M. Measurement-based quantum computation. Nat. Phys. 5, 19 (2009).
88. Ristè, D. et al. Deterministic entanglement of superconducting qubits by parity measurement and feedback. Nature 502, 350 (2013).

89. Ristè, D. et al. Detecting bit-flip errors in a logical qubit using stabilizer measurements. Nat. Commun. 6, 6983 (2015)

90. Córcoles, A. D. et al. Demonstration of a quantum error detection code using a square lattice of four superconducting qubits. Nat. Commun. 6, 6979 (2015).

91. Kelly, J. et al. State preservation by repetitive error detection in a superconducting quantum circuit. Nature 519, 66 (2015).

92. Blumoff, J. Z. et al. Implementing and characterizing precise multiqubit measurements. Phys. Rev. X 6, 31041 (2016).

93. Takita, M., Cross, A. W., Córcoles, A. D., Chow, J. M. \& Gambetta, J. M. Experimental demonstration of fault-tolerant state preparation with superconducting qubits. Phys. Rev. Lett. 119, 180501 (2017).

94. Gong, M. et al. Experimental verification of five-qubit quantum error correction with superconducting qubits. Preprint at https://arxiv.org/abs/1907.04507 (2019).

95. Kitaev, A. Y. Fault-tolerant quantum computation by anyons. Ann. Phys. 303, 2 (2003).

96. Zhu, G., Subaşı, Y., Whitfield, J. D. \& Hafezi, M. Hardware-efficient fermionic simulation with a cavity-QED system. npj Quantum Inf. 4, 16 (2018).

97. Vool, U. \& Devoret, M. Introduction to quantum electromagnetic circuits. Int. J. Circuit Theory Appl. 45, 897 (2017).

98. Nielsen, M. A. A simple formula for the average gate fidelity of a quantum dynamical operation. Phys. Lett. A 303, 249 (2002).

\section{ACKNOWLEDGEMENTS}

We thank Hannes Pichler and Nicolas Roch for useful discussions. This work was supported by the Army Research Laboratory Center for Distributed Quantum Information via the project SciNet and by the EU QuantEra project QTFLAG. A.S. is funded by the European Union's Horizon 2020 research and innovation program under grant agreement No. 714235. M.L.J. is funded by the European Union's Horizon 2020 research and innovation program under grant agreement No. 736943.

\section{AUTHOR CONTRIBUTIONS}

P.O.G., B.V., and P.Z. devised the project and carried out the analysis of the model. P. O.G, M.L.J., A.S., and G.K. developed the superconducting circuit implementation. All authors discussed the results and wrote the paper.

\section{COMPETING INTERESTS}

The authors declare no competing interests.

\section{ADDITIONAL INFORMATION}

Supplementary information is available for this paper at https://doi.org/10.1038/ s41534-020-0261-9.

Correspondence and requests for materials should be addressed to P.-O.G.

Reprints and permission information is available at http://www.nature.com/ reprints

Publisher's note Springer Nature remains neutral with regard to jurisdictional claims in published maps and institutional affiliations.

Open Access This article is licensed under a Creative Commons Attribution 4.0 International License, which permits use, sharing, adaptation, distribution and reproduction in any medium or format, as long as you give appropriate credit to the original author(s) and the source, provide a link to the Creative Commons license, and indicate if changes were made. The images or other third party material in this article are included in the article's Creative Commons license, unless indicated otherwise in a credit line to the material. If material is not included in the article's Creative Commons license and your intended use is not permitted by statutory regulation or exceeds the permitted use, you will need to obtain permission directly from the copyright holder. To view a copy of this license, visit http://creativecommons. org/licenses/by/4.0/.

(c) The Author(s) 2020 